\title{
STACKED WEAK LENSING MASS CALIBRATION: ESTIMATORS, SYSTEMATICS, AND IMPACT ON COSMOLOGICAL PARAMETER CONSTRAINTS
}

\author{
Eduardo Rozo ${ }^{1,2,5}$, HaO-Yi Wu ${ }^{3}$, and Fabian SchmidT ${ }^{4}$ \\ ${ }^{1}$ Department of Astronomy \& Astrophysics, The University of Chicago, Chicago, IL 60637, USA \\ ${ }^{2}$ Kavli Institute for Cosmological Physics, Chicago, IL 60637, USA \\ ${ }^{3}$ Kavli Institute for Particle Astrophysics and Cosmology, Physics Department, and \\ SLAC National Accelerator Laboratory, Stanford University, Stanford, CA 94305, USA \\ ${ }^{4}$ Theoretical Astrophysics, California Institute of Technology, M/C 350-17, Pasadena, California 91125, USA \\ Received 2010 September 14; accepted 2011 April 11; published 2011 June 24
}

\begin{abstract}
When extracting the weak lensing shear signal, one may employ either locally normalized or globally normalized shear estimators. The former is the standard approach when estimating cluster masses, while the latter is the more common method among peak finding efforts. While both approaches have identical signal-to-noise in the weak lensing limit, it is possible that higher order corrections or systematic considerations make one estimator preferable over the other. In this paper, we consider the efficacy of both estimators within the context of stacked weak lensing mass estimation in the Dark Energy Survey (DES). We find that the two estimators have nearly identical statistical precision, even after including higher order corrections, but that these corrections must be incorporated into the analysis to avoid observationally relevant biases in the recovered masses. We also demonstrate that finite bin-width effects may be significant if not properly accounted for, and that the two estimators exhibit different systematics, particularly with respect to contamination of the source catalog by foreground galaxies. Thus, the two estimators may be employed as a systematic cross-check of each other. Stacked weak lensing in the DES should allow for the mean mass of galaxy clusters to be calibrated to $\approx 2 \%$ precision (statistical only), which can improve the figure of merit of the DES cluster abundance experiment by a factor of $\sim 3$ relative to the self-calibration expectation. A companion paper investigates how the two types of estimators considered here impact weak lensing peak finding efforts.
\end{abstract}

Key words: cosmology: miscellaneous - galaxies: clusters: general

\section{INTRODUCTION}

Upcoming large photometric surveys such as the Dark Energy Survey (DES), ${ }^{6}$ Pan-Starrs, ${ }^{7}$ and the Large Synoptic Survey Telescope ${ }^{8}$ will find hundreds of thousands of clusters over large fractions of the sky. These samples hold the potential to be our most powerful tool for understanding dark energy (Albrecht et al. 2006) and appear to be a necessary component of any set of observables that wishes to distinguish between dark energy and modified gravity approaches for explaining an accelerating universe (Shapiro et al. 2010). Indeed, even today, cluster abundances provide some of the tightest constraints on the amplitude of the low-redshift matter power spectrum (Mantz et al. 2008; Henry et al. 2009; Vikhlinin et al. 2009; Rozo et al. 2010) and interesting constraints on modifications to gravity (Schmidt et al. 2009c; Rapetti et al. 2010; Lombriser et al. 2010). Not surprisingly then, realizing the promise of galaxy clusters as a cosmological probe is of paramount importance for understanding the physics driving the current phase of accelerated expansion of the universe.

The most important obstacle that cosmological applications of cluster surveys must overcome is the calibration of mass-observable relations. That is, the cold dark matter (CDM) paradigm of structure formation allows us to predict the abundance of galaxy clusters as a function of mass, whereas empirically we can only recover the abundance of galaxy clusters as a function of some observable $X$ that correlates with mass. Consequently, cosmological investigations of cluster abundances

\footnotetext{
5 Einstein Fellow.

6 http://www.darkenergysurvey.org/

7 http://pan-starrs.ifa.hawaii.edu/

8 http://www.lsst.org/
}

require that we carefully calibrate the probability $P(X \mid M)$ that a halo of mass $M$ is included in a survey as a cluster with observable $X$. This is a problem that is particularly difficult for photometrically selected cluster samples, as there is little theoretical understanding of the relation between a cluster's galaxy content and its total mass.

Self-calibration is an elegant attempt to overcome this difficulty. In this approach, one simply parameterizes $P(X \mid M)$, and fits for the corresponding parameters relying on clustering information (Lima \& Hu 2004, 2005; Hu \& Cohn 2006), and/or the evolution of abundances with redshift (Majumdar \& Mohr 2004; Gladders et al. 2007). One may further enhance such self-calibration techniques by relying on multiple mass tracers (Cunha 2009), and these approaches are expected to be very successful in improving our understanding of dark energy relative to other dark energy probes (Cunha et al. 2009). Nevertheless, it is expected that careful a priori calibration of the mass-observable relation of galaxy clusters from targeted follow-up observations can further enhance the utility of cluster samples over and above what can be achieved through self-calibration (Majumdar \& Mohr 2003, 2004; Wu et al. 2010). Moreover, the explicit assumptions made when performing self-calibration analysis (e.g., log-normal scatter) need to be empirically checked.

One way of empirically calibrating cluster masses is through cluster weak lensing stacking (Sealfon et al. 2006; Johnston et al. 2007a; Sheldon et al. 2009; Mandelbaum et al. 2008a; Leauthaud et al. 2010; White et al. 2010). This technique relies on coherently adding the weak lensing signal of galaxy clusters at fixed observables in order to estimate the mean mass of the stacked galaxy clusters. Relative to estimating the weak lensing mass of individual clusters, cluster stacking has the 
significant advantage of allowing us to detect the lensing signal at significantly lower cluster masses than would otherwise be possible. Moreover, averaging over many halos dramatically reduces the impact of weak lensing projection effects due to non-correlated structures along the line of sight. While such measurements by themselves do not in any way constrain the scatter of the observable-mass relation, the effectiveness of cluster stacking on improving cosmological constraints from photometrically selected cluster samples is evidenced by the dramatic improvement that this measurement produced on the cosmological constraints derived from the SDSS maxBCG cluster sample (Rozo et al. 2007, 2010). Indeed, one of the main results of this paper will be to demonstrate the impact that stacked weak lensing can have on cosmological constraints from future surveys (see also Oguri \& Takada 2011 for a similar analysis).

Here, we forecast the precision with which cluster mass and concentration can be measured in a DES-like photometric survey, and we discuss a variety of systematics associated with this measurement. Specifically, motivated by the large magnification bias signal expected in the weak lensing power spectrum in the DES (Schmidt et al. 2009a), we revisit the question of whether magnification bias can have a significant impact on weak lensing mass calibration (Mandelbaum et al. 2005). We also consider finite bin-width correction to density contrast estimates, and finally, we discuss how photometric redshift source selection can impact the recovered weak lensing signal.

This paper is one of two companion papers; the second paper (Schmidt \& Rozo 2011) considers the problem of identifying weak lensing peaks in large photometric surveys and the impact of magnification bias on weak lensing peak finding. An interesting by-product of having performed a simultaneous study of these two distinct subjects was the realization that in attacking these problems, different approaches for extracting the weak lensing signal are usually taken. Specifically, when estimating weak lensing masses one often bins galaxies in annuli and estimates the mean shear in an annulus by dividing by the number of galaxies found within the annulus, in other words, adopting a local normalization. Peak finders, on the other hand, often estimate the shear signal within a region by simply filtering the shear map that the galaxies sample without necessarily dividing by the number of galaxies within the filtered region. In other words, they employ a global normalization, one that does not depend on the local galaxy density. In these papers, we explore how each of these choices of normalization can impact the statistics and systematics of both peak finding and mass estimation.

This paper is organized as follows. In Section 2, we introduce the weak lensing estimator and calculate the statistical uncertainties of cluster mass obtained from this estimator. Various systematic errors are addressed in Section 3. We study the impact of these mass estimates on the cosmological parameter constraints in Section 4. Finally, we conclude in Section 5.

We adopt a fiducial flat $\Lambda$ CDM cosmology with $\Omega_{m}=0.28$, $\Omega_{\Lambda}=0.72, h=0.7, \Delta \zeta=4.54 \times 10^{-5}\left(\sigma_{8}=0.81\right)$, and $n_{s}=0.96$. All masses are $M_{200 m},{ }^{9}$ and distances are physical distances in either $\mathrm{kpc}$ or Mpc (as opposed to $h^{-1} \mathrm{kpc}$ or $h^{-1} \mathrm{Mpc}$ ). Finally, we set the magnification bias parameter $q=1.5$ (see below for details).

\footnotetext{
9 That is, 200 overdensity with respect to the mean matter density.
}

\section{MASS CALIBRATION IN LARGE OPTICAL SURVEYS VIA CLUSTER STACKING}

\subsection{The Weak Lensing Shear Estimator}

We consider a weak lensing shear estimator of the form

$$
\widehat{\Delta \Sigma}=\frac{1}{\bar{n} A} \sum_{i} \Sigma_{c}\left(z_{i}\right) e_{i}
$$

Here, the sum is over all galaxies within some annulus of radius $R$, where $e_{i}$ is the tangential ellipticity of galaxy $i, \bar{n}$ is the mean density of galaxies, $A$ is the area of the annulus, and $\Sigma_{c}$ is the lensing critical surface density. We emphasize that throughout this section, we ignore both foreground and cluster membership contamination of the source galaxy population. We return to this problem in Section 3.4.

Expert readers will immediately discern that the estimator $\widehat{\Delta \Sigma}$ is different from the standard weak lensing shear estimator:

$$
\widehat{\Delta \Sigma}^{\prime}=\frac{1}{N} \sum_{i} \Sigma_{c}\left(z_{i}\right) e_{i}
$$

where $N$ is the total number of source galaxies within the annulus. For the next few sections, we will focus primarily on $\widehat{\Delta \Sigma}$ rather than $\widehat{\Delta \Sigma}^{\prime}$. This is primarily for convenience: the fact that the estimator $\widehat{\Delta \Sigma}^{\prime}$ takes the form $\widehat{\Delta \Sigma}^{\prime}=x / y$ where $x$ and $y$ are correlated implies that computing its mean and variance requires some additional algebraic gymnastics that we need not worry about when considering $\widehat{\Delta \Sigma}$. Consequently, we have opted to illustrate our discussion with $\widehat{\Delta \Sigma}$ first and then treat the more complicated case of $\widehat{\Delta \Sigma}^{\prime}$. Section 2.5 discusses how the two estimators compare in terms of statistical precision, while in Section 3.4 we demonstrate that the two estimators have significantly different systematics with respect to foreground contamination of the source galaxy population.

In is worth noting at this point that works that have relied on stacked weak lensing (e.g., Sheldon et al. 2009; Mandelbaum et al. 2008b) have in fact been relying on the globally normalized estimator from Equation (1). This might seem surprising, since the starting point for both of the analyses just mentioned is a locally normalized estimator. We note, however, that in both analyses their locally normalized estimator is ultimately multiplied by the ratio of the number of galaxies around sources over the number of galaxies about random points. This effectively cancels out the $1 / N$ denominator from the local estimator, and replaces it by $1 / \bar{n} A$, reducing the estimator to the global one. In both cases, this procedure is introduced as a way of correcting for source dilution, which, as we demonstrate below, is one of the reasons why globally normalized estimators are potentially very useful. In summary, there is some sense in which the work here is a recasting of the systematic treatment in the Sheldon et al. (2009) and Mandelbaum et al. (2008b) works. That said, we believe that the realization that our locally and globally normalized estimators are different, particularly with respect to their systematic uncertainty, is a new one. Moreover, this realization paves the way for additional work, such as the possibility of joint shear and magnification analysis, which has the potential to self-calibrate systematic uncertainties (Rozo \& Schmidt 2010; Vallinotto et al. 2010).

We wish to estimate the mean and variance of $\widehat{\Delta \Sigma}$. In the interest of simplicity, we assume all sources are at the same redshift $z$ and set $\Sigma_{c}\left(z_{i}\right)=\bar{\Sigma}_{c}$. It is also useful to rewrite our 
estimator as follows: first, we pixelize the sky behind the lens in pixels of area $\Delta \Omega$, and define $N_{\alpha}$ as the number of galaxies in pixel $\alpha$. One might naively expect

$$
N_{\alpha}=\bar{n} \Delta \Omega\left(1+\delta_{\alpha}\right)
$$

where the $\delta_{\alpha}$ is the overdensity of matter in pixel $\alpha$. In practice, however, lensing modifies the observed source galaxy density such that (Schmidt et al. 2009b)

$$
N_{\alpha}=\bar{n} \Delta \Omega \mu_{\alpha}^{q / 2}\left(1+\delta_{\alpha}\right),
$$

where $\mu_{\alpha}$ is the magnification evaluated at pixel $\alpha$ and $q$ is a number that characterizes how the source density changes due to gravitational lensing. The magnification is related to the shear and convergence via $\mu^{-1}=(1-\kappa)^{2}-\gamma^{2}$, while the parameter $q$ may be positive or negative, depending on whether the increase in galaxy density due to the magnitude and size boosting of galaxy sources dominates over the skydilution effect of magnification. For a DES-like survey, we expect $q \approx 1-2$ (Schmidt et al. 2009b). Defining the filter function $W_{i}$ such that $W_{\alpha}=1$ only when a pixel is within the annulus of interest, and assuming the pixels are small enough that $N_{\alpha}$ is always either zero or one, we rewrite $\widehat{\Delta \Sigma}$ as

$$
\widehat{\Delta \Sigma}=\frac{\bar{\Sigma}_{c}}{A} \sum_{\alpha} \Delta \Omega \mu_{\alpha}^{q / 2}\left(1+\delta_{\alpha}\right) W_{\alpha} e_{\alpha},
$$

where the sum is now over all pixels. This is the equation we will use to derive the expectation value and variance of $\widehat{\Delta \Sigma}$.

\subsection{Mean and Variance of $\widehat{\Delta \Sigma}$}

The expectation value of $\widehat{\Delta \Sigma}$ follows from direct substitution of the expectation values of $e_{\alpha}$ and $\delta_{\alpha}$. Specifically,

$$
\begin{aligned}
& \left\langle e_{\alpha}\right\rangle=g_{\alpha} \\
& \left\langle\delta_{\alpha}\right\rangle=0,
\end{aligned}
$$

where $g$ is the reduced shear, $g=\gamma /(1-\kappa)$. The exact relation between tangential ellipticity and reduced shear $g$ depends on the adopted definition of ellipticity, and the responsivity of the sources to gravitational shear, for instance through polarizability. In the interest of simplicity, we rely on Equation (6), and simply note that in practice one needs to properly account for these additional complication (see e.g., Schneider \& Seitz 1995, for a discussion of how shear impacts a galaxy's observed ellipticity). We assume now that we work in the thin annulus approximation so that the variation of $\mu$ and $g$ within an annulus is negligible (though see also Section 3.3), and find

$$
\langle\widehat{\Delta \Sigma}\rangle=\bar{\Sigma}_{c} \mu^{q / 2} g \approx \Sigma_{c} \gamma,
$$

where all lensing quantities are to be evaluated at the annulus radius of interest. The approximate equality is obtained by expanding to leading order in the lensing quantities.

To compute the variance, we assume source galaxies are unclustered, so that $N_{\alpha}$ is Poisson. We have then

$$
\operatorname{Cov}\left(N_{\alpha}, N_{\beta}\right)=\delta_{\alpha \beta} \operatorname{Var}\left(N_{\alpha}\right)=\delta_{\alpha \beta}\left\langle N_{\alpha}\right\rangle=\delta_{\alpha \beta} \mu^{q / 2} \bar{n} \Delta \Omega,
$$

and therefore

$$
\left\langle\delta_{\alpha} \delta_{\beta}\right\rangle=\frac{\operatorname{Cov}\left(N_{\alpha}, N_{\beta}\right)}{\left\langle N_{\alpha}\right\rangle\left\langle N_{\beta}\right\rangle}=\delta_{\alpha \beta} \frac{1}{\mu^{q / 2} \bar{n} \Delta \Omega} .
$$

In Appendix A, we consider the additional contribution to the noise due to clustering of the source population and we demonstrate that it is sub-dominant at high masses, and negligible at low masses, justifying the assumption above. We further assume the variance in the ellipticity of galaxies is dominated by shape noise,

$$
\left\langle e_{\alpha} e_{\beta}\right\rangle=g_{\alpha} g_{\beta}+\delta_{\alpha \beta} \frac{\sigma_{e}^{2}}{2},
$$

the factor of two coming from the fact that there are two independent ellipticity components and we use only one of them, so $\sigma_{e}$ is the variance in the magnitude of the ellipticity, not the variance of each individual component. For quantitative purposes, we adopt $\sigma_{e}=0.3$ as our fiducial value for the amplitude of the shape noise term, which is somewhat smaller than the $0.35=\sqrt{2} \times 0.25$ value adopted by the Dark Energy Task Force (Albrecht et al. 2006). This will somewhat overestimate the relative importance of systematic uncertainties in this study, though we note that the scaling of our errors with shape noise is trivial, and our cosmological forecast does consider the impact of reducing the source density by half, which is equivalent to increasing shape noise by $\sqrt{2}$, which would be larger than the Albrecht et al. (2006) value. Direct substitution results in

$$
\operatorname{Var}(\widehat{\Delta \Sigma})=\bar{\Sigma}_{c} \frac{2 \mu^{q / 2}}{\bar{n} A}\left(g^{2}+\frac{1}{2} \sigma_{e}^{2}\right) \approx \bar{\Sigma}_{c}^{2} \frac{\sigma_{e}^{2}}{2 \bar{n} A}
$$

where again the approximate equality gives the leading order term in a series expansion of the lensing quantities.

The signal-to-noise ratio $(\mathrm{S} / \mathrm{N})$ of our estimator $\widehat{\Delta \Sigma}$ is

$$
(\mathrm{S} / \mathrm{N})=\mu^{q / 4} \frac{(\bar{n} A)^{1 / 2} g}{\left(g^{2}+\sigma_{e}^{2} / 2\right)^{1 / 2}} \approx \frac{(\bar{n} A)^{1 / 2} \gamma}{\left(\sigma_{e}^{2} / 2\right)^{1 / 2}} .
$$

In a DES-like survey, we expect $q \approx 1-2$, in which case the magnification bias correction $\mu^{q / 4}$ results in a modest increase of the $\mathrm{S} / \mathrm{N}$ of our measurement.

\subsection{Survey Assumptions, Fiducial Model, and the Fisher Matrix}

We wish to estimate the precision with which stacked shear weak lensing experiments can constrain halo mass and concentration in a DES-like survey. We adopt a survey area $\Omega=$

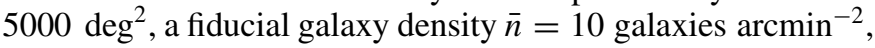
and a source redshift distribution

$$
f(z) \propto z^{m} \exp \left[-\left(z / z_{*}\right)^{\beta}\right]
$$

with $z_{*}=0.5, m=2$, and $\beta=1.4$, as appropriate for a DES-like survey (J. Annis 2009, private communication). The mean source redshift corresponding to this distribution is $\langle z\rangle=0.82$. The fraction of galaxies above a given redshift $z$ is given by

$$
F_{\mathrm{bg}}(z)=\frac{\int_{z}^{\infty} d z^{\prime} f\left(z^{\prime}\right)}{\int_{0}^{\infty} d z^{\prime} f\left(z^{\prime}\right)} .
$$

Given a lens redshift $z_{L}$, the (approximate) effective source density is simply $\bar{n} F_{\mathrm{bg}}\left(z_{L}\right)$. For a characteristic lens redshift $z_{L} \approx 0.5$, the corresponding source density is $\approx 8$ galaxies $\mathrm{deg}^{-2}$. 
We further assume clusters are binned in narrow redshift slices $z=\bar{z} \pm 0.05$, and are logarithmically binned in mass in bins of width $\pm \Delta \log _{10} M=0.1$, corresponding to 5 bins per decade in mass. The effective source density for a given mass and redshift bin is therefore

$$
\bar{n}_{\mathrm{eff}}=2 \bar{n} F_{\mathrm{bg}}(z) \frac{d n}{d \ln M} \Delta \ln M \Delta V,
$$

where the factor of two arises from the total width of the mass bin $2 \Delta \ln M, \Delta V$ is the survey volume enclosed by the redshift slice $z \pm \Delta z$

$$
\Delta V=(1+z)^{2} D_{A}^{2}(z) \Omega c H^{-1}(z) 2 \Delta z,
$$

$D_{A}$ being the angular diameter distance, and $F_{\mathrm{bg}}(z)$ is the fraction of source galaxies with redshift higher than $z$. When estimating all lensing properties, we will further assume that all source galaxies behind the clusters reside at a single source redshift $z_{s}$ equal to the mean source redshift of the sources behind the cluster. The mass function $d n / d \ln M$ is computed using the Tinker et al. (2008) mass function in our fiducial cosmology. As mentioned in the Introduction, for the purposes of computing the impact of magnification bias we always assume $q=1.5$ (Schmidt et al. 2009b).

Note that we have not taken into account the effects of finite mass bins here: since the scale radius of halos evolves with mass, the stacked shear profile within $[\ln M-\Delta \ln M ; \ln M+\Delta \ln M]$ is not equal to the profile of a Navarro-Frenk-White (NFW) halo with mass $M$. While this needs to be taken into account when fitting actual data, it is not of direct relevance to our Fisher forecast, so we neglect this effect here.

Furthermore, it should be noted that, observationally, cluster stacks are made by binning in an observable $X$, whereas the problem we have laid out here assumes that the clusters are binned in mass. This will have very little impact on the errors in the mass, as these are dominated by shape noise. It is only the interpretation of the recovered mass that would vary, which would represent the mean mass of all galaxy clusters within a bin. For reference, the intrinsic scatter of the maxBCG (Koester et al. 2007) galaxy clusters is $\approx 35 \%$ (Rozo et al. 2009a). The corresponding bias in the recovered masses is $0.5 \alpha \sigma^{2} \approx 20 \%$ where $\alpha$ is the slope of the halo mass function (see e.g., Mortonson et al. 2010), which is large but well understood, and therefore should have a minimal to negligible impact on the determination of the richness-mass relation, and therefore on our cosmological forecasts. Given our expectations and the fact that our Fisher matrix forecast ought to be interpreted as a rough estimate of the precision of this type of analysis, we have ignored this (mass proxy-dependent) complication. We also note that Oguri \& Takada (2011) reached very similar conclusions to ours having explicitly included this term, which is consistent with our expectations that this detail has little impact on our conclusions.

Assuming sources are uncorrelated (i.e., source galaxies are randomly distributed) and that the redshift slices are narrow enough that halos are non-overlapping, the estimators $\widehat{\Delta \Sigma}$ for different radial bins are uncorrelated. The non-overlapping halo assumption necessarily breaks down at sufficiently large scales. For instance, Sheldon et al. (2009) find that on $5 \mathrm{Mpc}$ scales, the weak lensing signal of galaxy clusters becomes correlated due to halo overlaps. Here, we will restrict ourselves to $2 \mathrm{Mpc}$ when estimating the mass uncertainty. Moreover, because clusters subtend smaller angles at higher redshift, this should be less of a problem for DES clusters than for maxBCG clusters. In this limit of uncorrelated radial bins, the Fisher matrix for a weak lensing shear experiment simplifies to ${ }^{10}$

$$
F_{\mathrm{ab}}=\sum_{\alpha} \frac{1}{\operatorname{Var}\left(\Delta \Sigma_{\alpha}\right)} \frac{\partial\left\langle\widehat{\Delta \Sigma}_{\alpha}\right\rangle}{\partial p_{a}} \frac{\partial\left\langle\widehat{\Delta \Sigma}_{\alpha}\right\rangle}{\partial p_{b}},
$$

where the sum is over all radial bins $\alpha$ and $\boldsymbol{p}$ is the vector of parameters of interest. Throughout, we assume logarithmic radial binning with bins of width $\pm \Delta \log _{10} R=0.02$. Moreover, we will only add bins over the radial range $0.1-2 \mathrm{Mpc}$. We will further impose the condition that the cluster magnification must be smaller than five (i.e., $|\mu| \leqslant 5$ ) for a radial bin to be included in our computation. This ensures that for those systems where the Einstein radius extends past the $0.1 \mathrm{Mpc}$ minimum radius, we do not include information from sources that are strongly lensed. Our results are robust to making our bins narrower, but do depend in detail on the radial range assumed. For a discussion, see Section 3.2.

We model the mass distribution of cluster stacks as a NFW(Navarro et al. 1996) profile. For simplicity, we have held the concentration parameter of our clusters fixed to $c=5$. Changes in concentration within a stack do not qualitatively change any of our results, and have only a modest impact on the quantitative results. All of our conclusions are robust to the choice of concentration parameter. We compute the convergence and shear of NFW profiles using the formulae in Wright \& Brainerd (2000; see also Bartelmann 1996 and the appendices of Schmidt \& Rozo 2011).

\subsection{Results}

Figure 1 shows the predicted statistical uncertainty in the weak lensing mass and concentration of our cluster stacks as a function of the mean cluster mass of the stack. Different lines correspond to different lens redshift. From top to bottom, the solid lines assume lens redshifts $z_{\text {lens }}=0.1,0.2$, and 0.4 . From bottom to top, the dashed lines assume $z_{\text {lens }}=0.6,0.9$, and 1.2. The vertical dotted line is a rough estimate of the expected mass threshold for optical selection. Qualitatively, the precision of weak lensing measurements increases from $z=0$ to $z \approx 0.2$, reflecting the increasing number of lenses due to increased survey volume, as well as the improvement on the lensing efficiency of the lenses. Between $z \approx 0.2$ and $z \approx 0.6$, the precision of the weak lensing measurements is roughly redshift independent: even though survey volume continues to increase, it is now offset by a diminishing source density. For redshifts $z \gtrsim 0.6$, both the lensing efficiency and the effective source density decrease quickly with increasing lens redshift, so weak lensing masses begin to worsen. We also note that at all redshifts there is a mass scale at which the errors blow up, reflecting the exponential drop off in the halo mass function. The typical precision with which the mean mass of galaxy clusters may be estimated at moderate redshifts is roughly $2 \%$.

\footnotetext{
${ }^{10}$ In the interest of simplicity, in Equation (18) we have neglected the modicum of information in the small dependence of $\operatorname{Var}(\widehat{\Delta \Sigma})$ on the model parameters $\boldsymbol{p}$. We do not expect our results to be sensitive to this detail. Moreover, in practice we expect the covariance matrix for the measurements will be estimated using a jackknife method, which will erase any information in the variation of the covariance matrix with $p$.
} 

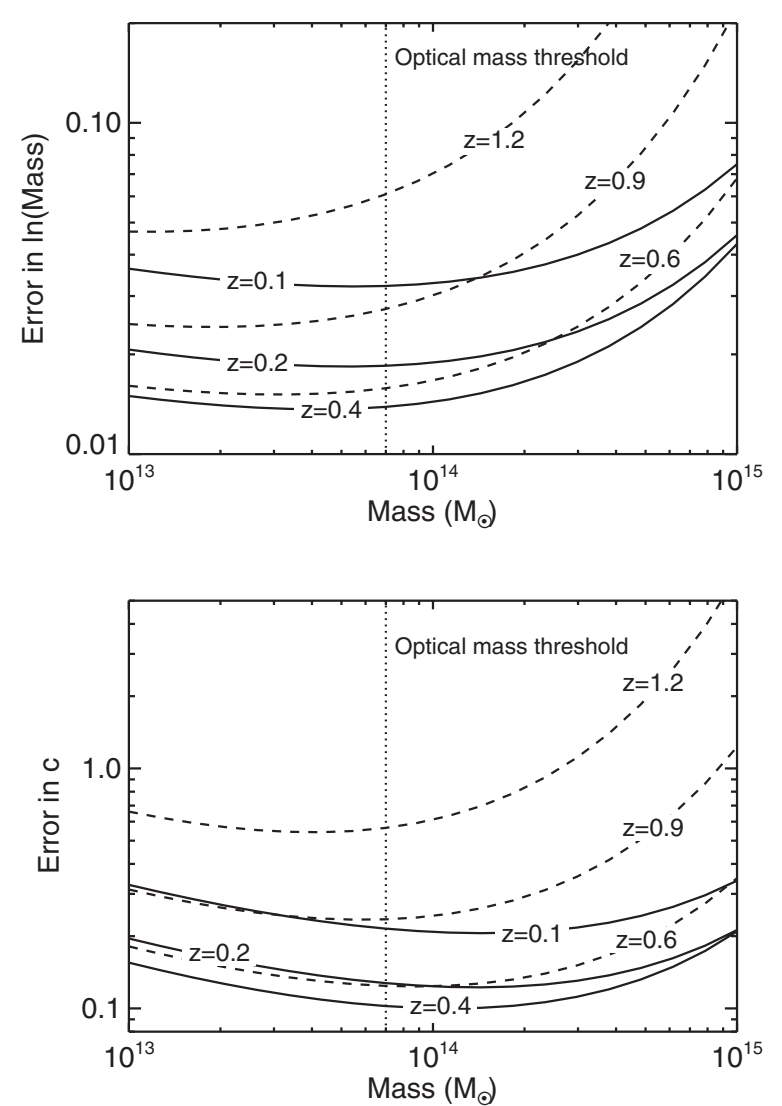

Figure 1. Predicted $1 \sigma$ statistical error in the log-mass (top) and the concentration (bottom) of galaxy clusters in a DES-like survey as measured through cluster stacking. The solid lines illustrate the trends with redshift for $z \leqslant 0.4$ while the dashed lines illustrate the trends with redshift for $z \geqslant 0.4$. From top to bottom (in either plot) the lens redshifts of the solid curves are $z_{\text {lens }}=0.1,0.2$, 0.4 , whereas the redshift of the dashed curves is, from bottom to top, $z_{\text {lens }}=0.6$, 0.9 , and 1.2. The typical precision with which the mean mass of galaxy clusters may be estimated at moderate redshifts is roughly $2 \%$.

\subsection{On the Choice of Estimator}

In Section 2.1, we defined the estimator $\widehat{\Delta \Sigma}$ differently from the more commonly used weak lensing shear estimator:

$$
\widehat{\Delta \Sigma}^{\prime}=\frac{1}{N} \sum_{i} \Sigma_{c}\left(z_{i}\right) e_{i}
$$

Pixelizing the sky, the estimator $\widehat{\Delta \Sigma}^{\prime}$ can be written as

$$
\widehat{\Delta \Sigma}^{\prime}=\bar{\Sigma}_{c} \frac{\sum_{\alpha} \Delta \Omega \mu_{\alpha}^{q / 2}\left(1+\delta_{\alpha}\right) W_{\alpha} e_{\alpha}}{\sum_{\alpha} \Delta \Omega \mu_{\alpha}^{q / 2}\left(1+\delta_{\alpha}\right) W_{\alpha}},
$$

where the sum is now over all pixels.

A difficulty with $\widehat{\Delta \Sigma}^{\prime}$ is now immediately apparent. We see that $\widehat{\Delta \Sigma}^{\prime}$ takes the form $\widehat{\Delta \Sigma}^{\prime}=x / y$, and in general one has $\left\langle\widehat{\Delta \Sigma}^{\prime}\right\rangle=\langle x / y\rangle \neq\langle x\rangle /\langle y\rangle$. One way to address this problem is to write $y=\langle y\rangle+\delta y$ and expand the denominator in a power series using the binomial expansion. This results in a power series expansion of all quantities in terms of $1 /(\bar{n} A)$ (see Appendix B). Doing so up to second order in $\delta$ to compute the mean of $\widehat{\Delta \Sigma}^{\prime}$ we find

$$
\left\langle\widehat{\Delta \Sigma}^{\prime}\right\rangle=\bar{\Sigma}_{c} \frac{\left\langle\mu^{q / 2} g\right\rangle}{\left\langle\mu^{q / 2}\right\rangle} .
$$

The quantities in the angular brackets in the right hand side of the equation are to be averaged over the radial bin of interest. Adopting the thin annulus approximation, we can pull out the $\mu_{i}^{q / 2}$ term out of the sums in Equation (20) to obtain

$$
\widehat{\Delta \Sigma}^{\prime}=\overline{\Sigma_{c}} \frac{\sum_{\alpha} \Delta \Omega\left(1+\delta_{\alpha}\right) e_{\alpha}}{\sum_{\alpha} \Delta \Omega\left(1+\delta_{\alpha}\right)} .
$$

In this limit, the magnification term drops entirely out of the equations, and one can easily solve for the mean and variance of $\widehat{\Delta \Sigma}^{\prime}$ using the power series approach advocated earlier. We find (see Appendix B)

$$
\begin{gathered}
\left\langle\widehat{\Delta \Sigma}^{\prime}\right\rangle=\bar{\Sigma}_{c} g \\
\operatorname{Var}\left(\widehat{\Delta \Sigma}^{\prime}\right)=\bar{\Sigma}_{c}^{2} \frac{1}{\mu^{q / 2} \bar{n} A} \frac{\sigma_{e}^{2}}{2} .
\end{gathered}
$$

Note that the magnification still appears in the variance since the effective source density is $\mu^{q / 2} \bar{n}$.

Using the above equations, we repeat the Fisher matrix experiment that we carried out for $\widehat{\Delta \Sigma}$. We find that these two estimators recover the mean cluster mass of a stack with nearly identical precision, with $\left|\sigma_{M}-\sigma_{M^{\prime}}\right| \lesssim 0.2 \%$ where $\sigma_{M}$ and $\sigma_{M^{\prime}}$ are the forecasted uncertainties in $\langle\ln M\rangle$ for a stack using the estimators $\widehat{\Delta \Sigma}$ and $\widehat{\Delta \Sigma}^{\prime}$, respectively.

Before we end, we would like to issue a word of warning concerning the validity of our results for the estimator $\widehat{\Delta \Sigma}^{\prime}$. Our derivation in this section deals with the covariance between the numerator and denominator of $\widehat{\Delta \Sigma}^{\prime}$ through a power series expansion, where the expansion parameter is $1 / \bar{n} A$, the expected number of galaxies within the annulus of interest. Consequently, for our analysis to be applicable one must choose radial bins that are large enough for $\bar{n} A \gg 1$. On the other hand, we are also using the thin annulus approximation, so $A$ cannot be arbitrarily large. While we expect to be able to simultaneously satisfy both of these constraints in stacked weak lensing analysis, this is generally not possible for individual clusters. For instance, in our fiducial model, a relatively broad radial bin $R \in$ $[0.2 \mathrm{Mpc}, 0.4 \mathrm{Mpc}]$ would only contain $\approx 25$ galaxies, so the higher-order terms in the $1 / \bar{n} A$ power series expansion can in principle introduce $\approx 5 \%$ level corrections to the signal in the inner most radial bins. Here, we do not address this additional difficulty since our primary interest is stacked weak lensing. The finite width-bin corrections that we treat below concern corrections to the leading order term which occur even when $\bar{n} A \gg 1$.

\section{SYSTEMATICS}

\subsection{Magnification Bias Corrections}

By magnification bias corrections we refer to the terms that scale as $\mu^{q / N}$ for some value of $N$ in both the mean and variance of our shear estimators. As discussed in Section 2.5, in the thin annulus approximation the mean of $\widehat{\Delta \Sigma}^{\prime}$ is independent of these magnification bias terms, so we expect magnification bias corrections to be small. ${ }^{11}$ The same is not true of $\widehat{\Delta \Sigma}$,

\footnotetext{
${ }^{11}$ In principle, the fact that magnification bias does impact the covariance matrix of $\widehat{\Delta \Sigma}^{\prime}$ could lead to biasing in halo mass and concentration. In practice, however, the error bars employed when relying on $\widehat{\Delta \Sigma}^{\prime}$ are based on the Poisson variance of the observed number density, which includes the appropriate magnification bias. Thus, one does not expect $\widehat{\Delta \Sigma}^{\prime}$ to be affected by magnification bias.
} 


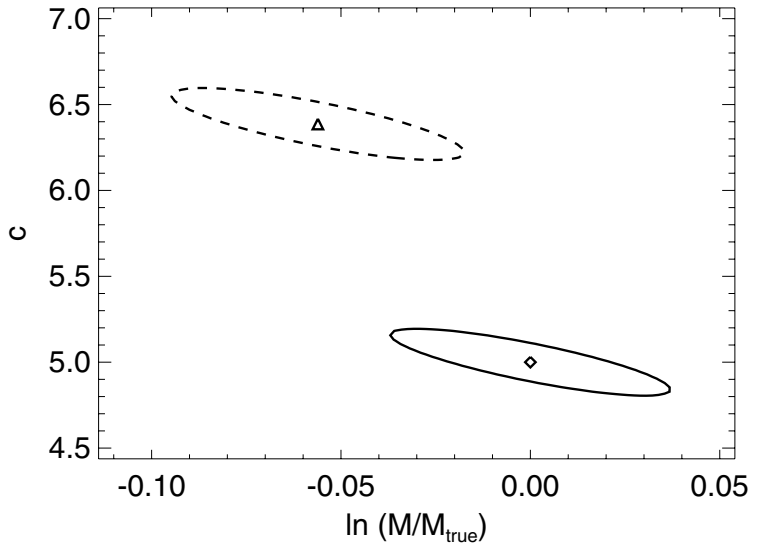

Figure 2. Sixty-eight percent likelihood contours obtained when measuring the mass and concentration of a halo stack of mass $M=5 \times 10^{14} M_{\odot}$ at redshift $z=0.3$ using a DES-like survey. The solid contour includes the corrections for magnification bias, while the dashed line ignores these corrections. The best-fit value for the former case coincides with the input parameters, marked by a diamond, while ignoring these corrections leads to biased expectation values, shown here with a triangle. This behavior is generic (see Figure 3 for details).

and therefore ignoring these corrections can potentially lead to systematic biases. That said, it is worth emphasizing that incorporating these corrections into the analysis is not difficult, so having to include such corrections is not any sort of limiting systematic. Our goal here is simply to demonstrate that doing so is a necessary step in the analysis of future data sets.

To address this question, we use the Fisher matrix formalism set out in the appendix of Wu et al. (2008; see also Huterer \& Linder 2007) to estimate the mass and concentration parameters that would be recovered from the data if magnification bias corrections are ignored, and then compare those results to those derived when properly including the magnification bias terms in the analysis. The basic idea is illustrated in Figure 2. The solid and dashed ellipses represent the $68 \%$ confidence contours when including (solid) or ignoring (dashed) magnification bias corrections for a halo stack at $z=0.3$ and $M=5 \times 10^{14} M_{\odot}$. The true halo mass and concentration is marked by the diamond while the triangle marks the best-fit halo mass and concentration obtained when ignoring magnification bias corrections. We can see that the best-fit values when ignoring magnification bias effects are well beyond the $68 \%$ error ellipse of the experiment, demonstrating that magnification bias is a significant correction when using the $\widehat{\Delta \Sigma}$ estimator.

The extent to which magnification bias is important relative to statistical uncertainties depends on both halo mass and redshift. In particular, magnification bias is more important at high masses, and at redshifts $z \approx 0.2-0.5$, for which lensing efficiency is high and statistical errors are small due to high source densities. This is illustrated in Figure 3, where we show the ratio between the systematic bias in mass and concentration incurred by ignoring magnification bias effects, relative to the corresponding statistical uncertainty. We see that our projected bias in the halo mass can be twice as large as the corresponding statistical uncertainty, while the corresponding errors in concentration can be even larger than 10 times the statistical error. Thus, inclusion of magnification bias and reduced shear corrections are necessary for weak lensing mass calibrations in a DES-like survey.

One aspect of our results that is very surprising is that magnification bias can lead to systematic underestimates of the mass, despite the fact that lensing bias leads to an apparent
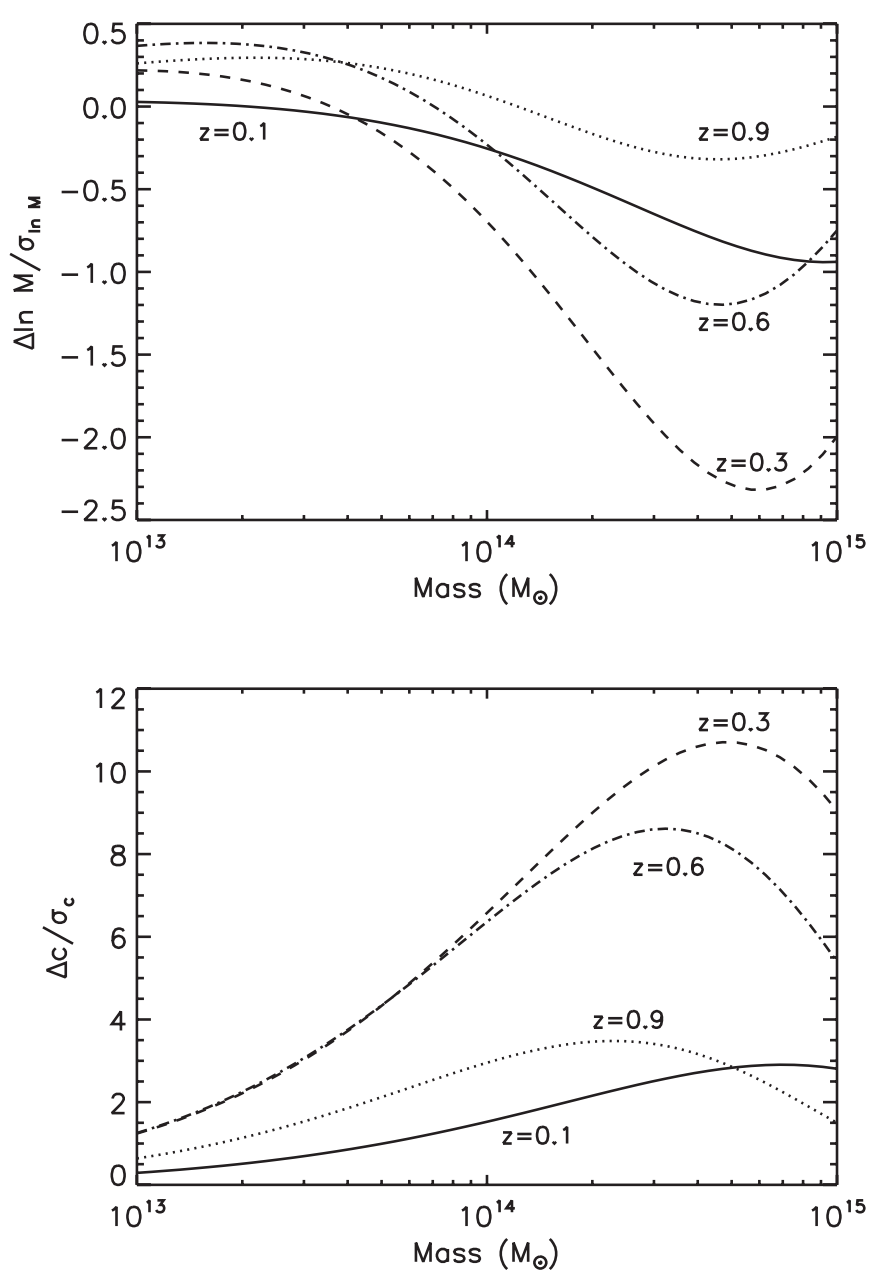

Figure 3. Ratio of the systematic bias in the log-mass $\Delta \ln M$ or concentration $\Delta c$ incurred when ignoring magnification bias and reduced shear errors, relative to the statistical uncertainty $\sigma_{\ln M}$ or $\sigma_{c}$ in these quantities (see Figure 1).

increase of the lensing signal. The reason we find that masses are underestimated when ignoring magnification bias is due to the anti-correlation between halo mass and concentration. This anti-correlation implies that random noise that leads to an increase in concentration typically leads to a decrease in mass. Here, the increase in concentration is systematics driven, but the effect is the same. This statement, however, is very much dependent on the range of radii considered in the calculation. In particular, as one moves out the maximum radius employed in the analysis the anti-correlation between mass and concentration decreases, and lensing bias begins to produce positively biased halo masses (see also Section 3.2).

Finally, our results may appear to contradict those in Schmidt \& Rozo (2011), who find magnification bias results in an overestimate of weak lensing masses despite the fact that they estimate masses using filters of comparable radius to those employed here. The reason for this apparent discrepancy is that in this analysis we have fit for both halo mass and concentration, whereas in the Schmidt \& Rozo (2011) analysis, the concentration parameter is held fixed at its fiducial value. In that case, the apparent increase in the matter density in the inner regions of a cluster due to magnification bias necessarily goes into halo mass rather than concentration, leading to a positive mass bias. In practice, because cluster stacks have abundant signal, one fits for both mass and concentration, so the observationally relevant case for cluster stacks is that considered 


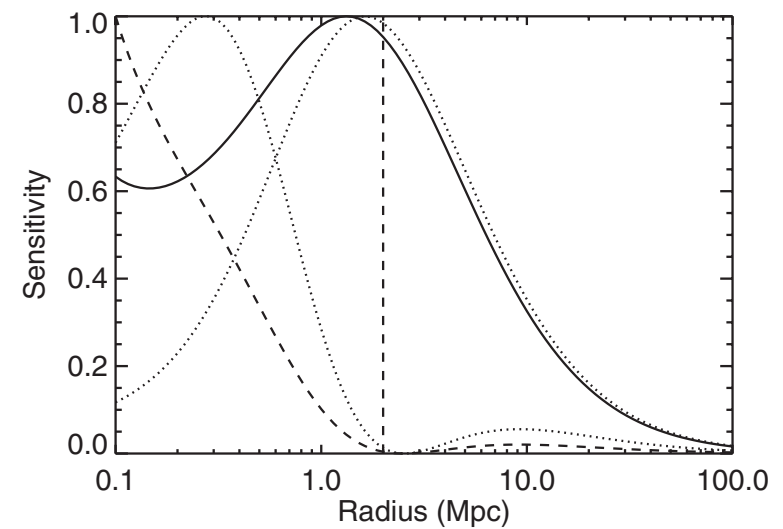

Figure 4. Mass (solid) and concentration (dashed) sensitivity as a function of radius for a $10^{15} M_{\odot}$ halo at redshift $z=0.6$. The sensitivity functions characterize the relative information content of various scales. Dotted curves are the two sensitivity functions we obtain when neglecting magnification bias corrections. Not surprisingly, the scales that dominate the concentration and mass information content are a few hundred $\mathrm{kpc}$ and a few $\mathrm{Mpc}$, respectively. In both cases, magnification bias shifts the sensitivity functions toward smaller scales, as we would expect. We emphasize that our mass estimates only include information from scales $R \leqslant 2 \mathrm{Mpc}$ (dashed lines), where the one-halo terms are expected to dominate. At large scales, our model is inappropriate, but it is nevertheless clear that there is significant amount of information that can be garnered by properly extending one's analysis to such large scales (see e.g., Oguri \& Takada 2011).

here. For individual weak lensing mass estimates in the low $\mathrm{S} / \mathrm{N}$ regime, the results of Schmidt \& Rozo (2011) are the relevant ones.

\subsection{The Relative Information Content of Different Scales}

In light of the results from the previous section it is worth investigating the contribution to the Fisher matrix from each individual radial bin as a function of radius. The information contributed by radial bin $R_{\alpha}$ is

$$
F_{\mathrm{ab}}\left(R_{\alpha}\right)=\frac{1}{\operatorname{Var}\left(\Delta \Sigma_{\alpha}\right)} \frac{\partial\left\langle\widehat{\Delta \Sigma}_{\alpha}\right\rangle}{\partial p_{a}} \frac{\partial\left\langle\widehat{\Delta \Sigma}_{\alpha}\right\rangle}{\partial p_{b}} .
$$

Of course, this quantity explicitly depends on the width of the radial bins employed. We therefore define the relative sensitivity $s_{\mathrm{ab}}$ via

$$
s_{\mathrm{ab}}\left(R_{\alpha}\right)=\frac{F_{\mathrm{ab}}\left(R_{\alpha}\right)}{\max \left\{F_{\mathrm{ab}}\left(R_{\alpha}\right)\right\}}
$$

assuming logarithmic radial bins. Note that, by construction, $s_{\mathrm{ab}}$ is very nearly independent of the assumed width of the radial bins and the assumed source density. Of particular interest to us are the mass sensitivity $s_{\mathrm{mm}}$ and concentration sensitivity $s_{\mathrm{cc}}$, which characterize the relative mass and concentration information content of various scales.

Figure 4 illustrates the mass (solid) and concentration (dashed) sensitivity functions for a $10^{15} M_{\odot}$ halo at redshift $z=0.6$. We note that the sensitivity functions here have not accounted for miscentering, membership contamination, nor intrinsic alignments, all of which will have an impact on the sensitivity functions. Note that even though when estimating masses we only include scales $R \leqslant 2 \mathrm{Mpc}$, we extend the radial range to $10^{2} \mathrm{Mpc}$ to enclose all radial scales that have significant information. Not surprisingly, the mass information content is dominated by scales of order a few Mpc, while the concentration information content is dominated by scales of order a few hundred kpc. The large overlap of the two sensitivity functions results in the anti-correlation between halo mass and concentration noted earlier. The dotted lines in the figure correspond to the sensitivity functions in the absence of magnification bias effects (i.e., setting $q=0$ ). We see that magnification bias shifts the sensitivity functions to smaller scales, effectively upweighting them, as we would expect. The corresponding sensitivity functions for the estimator $\widehat{\Delta \Sigma}^{\prime}$ are essentially identical to those of $\widehat{\Delta \Sigma}$ in the absence of magnification bias corrections.

In light of Figure 4 it is easy to understand why our results were sensitive to the adopted radial range. It is evident from the figure that both the minimum and maximum radius will impact our results. The minimum radius chosen will directly affect the precision of the concentration measurement, which in turn propagates to the cluster mass via the mass-concentration correlation. Likewise, it is easy to see why our results are sensitive to the maximum radius employed: scales as large as tens of Mpc still contain non-negligible amounts of information pertaining to the halo mass. Of course, in practice such scales show deviations from a simple projected NFW profile (Hoekstra 2003), both because of possible deviations from NFW in the one-halo term, and because of the appearance of a two-halo term. However, it is clear that there is significant additional information in the weak lensing signal up to scales as large as several tens of $\mathrm{Mpc}$, a more general treatment (e.g., Oguri \& Takada 2011) can make use of this signal.

\subsection{The Thin Annulus Approximation}

When performing our forecasts for both $\widehat{\Delta \Sigma}$ and $\widehat{\Delta \Sigma}^{\prime}$ we adopted the thin annulus approximation, in which one ignores variations of the convergence, shear, and magnification fields within an annulus. When using finite radial bin widths, however, there is a slight ambiguity on what radius should one employ when performing the thin annulus approximation. Two obvious choices are the central radius $R_{c}$ where the radial bins are defined by $\ln R_{c} \pm \Delta \ln R$, and the mean radius of the annulus

$$
\langle R\rangle=\frac{2}{3} \frac{R_{\max }^{3}-R_{\min }^{3}}{R_{\max }^{2}-R_{\min }^{2}}
$$

The question then becomes, how thin must an annulus be for the thin annulus approximation to hold for either of these two choices of radii?

To address this question, we have again relied on a Fisher matrix approach to estimate the systematic error in the logmass of a galaxy cluster when the mass is estimated using the thin-annulus approximation, as a function of the width of the annulus used to estimate $\widehat{\Delta \Sigma}$ or $\widehat{\Delta \Sigma}^{\prime}$. For specificity, we will focus here on $\widehat{\Delta \Sigma}^{\prime}$, but very similar conclusions hold for $\widehat{\Delta \Sigma}$. We emphasize that, just as with magnification bias corrections, one can in principle just include these finite bin-width effects in the fits. The analysis described here simply addresses whether it is necessary to do so.

Our results are shown in Figure 5. The largest bin width we consider is roughly five bins per decade in radius, corresponding to $\pm \Delta \log _{10} R=0.1$. For such a bin width, there is a negative mass bias of $\approx 5 \%$ assuming the thin annulus approximation at $R=R_{c}$ (solid line) and a $\approx 2 \%$ positive bias assuming $R=\langle R\rangle$ (dashed line). This bias is nearly independent of halo mass, concentration, redshift, or source galaxy density. Thus, it is important to account for finite bin-width effects 


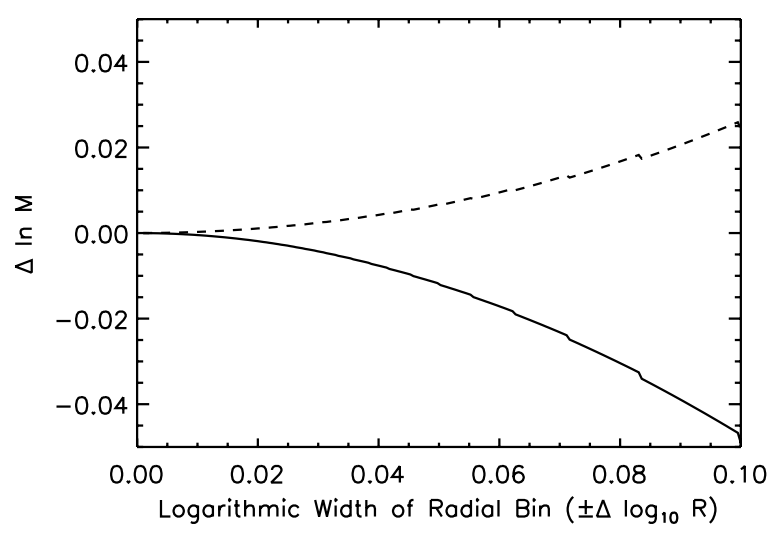

Figure 5. Bias in the recovered weak lensing masses using the thin-annulus approximation for predicting the expectation value of $\widehat{\Delta \Sigma}^{\prime}$ as a function of the width of the annulus used to estimate the shear profile. We assume $\widehat{\Delta \Sigma}^{\prime}$ is estimated in annuli of width $\Delta$ defined via $\left[\ln R_{c}-\Delta, \ln R_{c}+\Delta\right]$. The solid line assumes the thin annulus approximation at $R=R_{c}$, while the dashed line assumes the thin annulus approximation at the mean radius of the annulus $\langle R\rangle$. This bias is nearly independent of halo mass, concentration, redshift, and source density.

when estimating cluster masses, particularly for the purposes of calibrating cluster scaling relations. ${ }^{12}$

The origin of the above bias is simple to understand: first consider the case $R=R_{c}$. It is evident that in this case our annulus contains more area beyond $R=R_{c}$ than below $R=R_{c}$. Consequently, we expect a negative bias, since the outer region of the annulus is being upweighted relative to the inner region. Setting $R=\langle R\rangle$ evidently overcompensates for this effect. While one can in principle imagine finding the specific value of $R$ for which there is no bias, this value will depend in detail on cosmology, source galaxy density (due to magnification bias corrections), etc., so it is simplest to just take into account the finite bin-width correction exactly. Alternatively, when performing stacked weak lensing measurements one may adopt very narrow radial bins, in which case these corrections become negligible.

\subsection{Foreground and Cluster Member Dilution}

One important systematic when estimating the mass of galaxy clusters through weak lensing is the effect known as member dilution (Bernardeau 1998; Medezinski et al. 2007). That is, if cluster members or other foreground galaxies are included in the source population, they will reduce the observed shear and cause us to underestimate the cluster mass. Let us treat this problem explicitly: the total (angular) galaxy density around a cluster at redshift $z$ can be written as a sum

$$
n=n_{\mathrm{bg}}+n_{\mathrm{fg}}+n_{\mathrm{cl}},
$$

where $n_{\mathrm{bg}}$ is the background galaxy density field, $n_{\mathrm{fg}}$ is the foreground galaxy density, and $n_{\mathrm{cl}}$ is the density of cluster galaxies. When estimating weak lensing masses, one adopts photometric redshift cuts to preferentially select background galaxies. Let $r_{\mathrm{bg}}, r_{\mathrm{fg}}$, and $r_{\mathrm{cl}}$ be the probabilities of passing the photometric redshift cuts for a galaxy in the background, foreground, and at the lens redshift, respectively. A perfect photometric redshift selection would result in $r_{\mathrm{fg}}=0, r_{\mathrm{cl}}=0$,

12 The small "steps" that appear in Figure 5 are real. To estimate cluster masses, we use the scales $0.1 \mathrm{Mpc}<R<2 \mathrm{Mpc}$. This means that when we vary the bin width, there will be discrete jumps in the number of bins, which leads to the "stepping" observed in the plot. and $r_{\mathrm{bg}}=1$, so the value of these parameters characterizes the actual redshift distribution of the source sample in the presence of imperfect photometric redshift selection. Note that in practice these quantities will be functions of the source redshift population, but we are again relying on the single source approximation for simplicity. This is not realistic, but suffices for our purposes, namely demonstrating that $\widehat{\Delta \Sigma}$ and $\widehat{\Delta \Sigma}^{\prime}$ are affected by these systematics in different ways. For simplicity, we will work in the thin annulus limit throughout.

We begin by considering $\widehat{\Delta \Sigma}$, which we now write as

$$
\begin{aligned}
\Delta \Sigma= & \frac{\bar{\Sigma}_{c}}{\bar{n} A F} \sum_{i} \Delta \Omega\left[r_{\mathrm{bg}} n_{\mathrm{bg}} e_{i}^{\mathrm{bg}}+r_{\mathrm{fg}} n_{\mathrm{fg}} e_{i}^{\mathrm{fg}}\right] \\
& +\frac{\bar{\Sigma}_{c}}{\bar{n} A F} \sum_{i} \Delta \Omega n_{\mathrm{cl}} e_{i}^{\mathrm{cl}} .
\end{aligned}
$$

Here, $F$ is the fraction of galaxies with redshift higher than the redshift of the cluster (according to the observed redshift distribution), while $\bar{n}$ is the mean galaxy density over the whole survey. Hence, averaging over the whole survey,

$$
\left\langle n_{\mathrm{bg}}\right\rangle=F \bar{n} ; \quad\left\langle n_{\mathrm{fg}}\right\rangle=(1-F) \bar{n} .
$$

Further, $e^{\mathrm{fg}}, e^{\mathrm{bg}}$, and $e^{\mathrm{cl}}$ represent the foreground, background, and cluster ellipticity fields. The first two moments of $e^{\text {bg }}$ satisfy Equations (6) and (11), while for the foreground and cluster ellipticity fields we set

$$
\begin{gathered}
\left\langle e_{i}\right\rangle=0 \\
\left\langle e_{i} e_{j}\right\rangle=\frac{1}{2} \sigma_{e}^{2} \delta_{i j} .
\end{gathered}
$$

In practice, cluster member galaxies are known to be radially aligned (i.e., $\left\langle e_{i}^{\mathrm{cl}}\right\rangle \neq 0$ Faltenbacher et al. 2007), but we defer this additional source of error to future work. Upon plugging in, we find

$$
\langle\widehat{\Delta \Sigma}\rangle=r_{\mathrm{bg}} \bar{\Sigma}_{c} \mu^{q / 2} g
$$

implying that $\widehat{\Delta \Sigma}$ is sensitive to only one of the three systematic parameters considered here. The relative systematic error due to an uncertainty $\delta r_{\mathrm{bg}}$ in $r_{\mathrm{bg}}$ is simply

$$
\sigma_{\text {sys }}(\ln \Delta \Sigma)=\frac{\delta r_{\mathrm{bg}}}{r_{\mathrm{bg}}}=\delta r_{\mathrm{bg}}
$$

assuming our fiducial value $r_{\mathrm{bg}}=1$.

Let us now turn our attention to $\widehat{\Delta \Sigma}^{\prime}$, which takes the form

$$
\widehat{\Delta \Sigma}^{\prime}=\bar{\Sigma}_{c} \frac{x}{y}
$$

where

$$
\begin{gathered}
x=\sum_{i} \Delta \Omega\left[r_{\mathrm{bg}} n_{\mathrm{bg}} e_{i}^{\mathrm{bg}}+r_{\mathrm{fg}} n_{\mathrm{fg}} e_{i}^{\mathrm{fg}}+r_{\mathrm{cl}} n_{\mathrm{cl}} e_{i}^{\mathrm{cl}}\right] \\
y=\sum_{i} \Delta \Omega\left[r_{\mathrm{bg}} n_{\mathrm{bg}}+r_{\mathrm{fg}} n_{\mathrm{fg}}+r_{\mathrm{cl}} n_{\mathrm{cl}}\right] .
\end{gathered}
$$

We will again ignore correction terms to the mean that go as $1 /(\bar{n} A)$ and set $\left\langle\widehat{\Delta \Sigma}^{\prime}\right\rangle=\langle x\rangle /\langle y\rangle$. We then arrive at

$$
\left\langle\widehat{\Delta \Sigma}^{\prime}\right\rangle=\bar{\Sigma}_{c} g\left[1+\frac{r_{\mathrm{fg}}}{r_{\mathrm{bg}}} \frac{1-F}{\mu^{q / 2} F}+\frac{r_{\mathrm{cl}}}{r_{\mathrm{bg}}} \frac{\left\langle n_{\mathrm{cl}}\right\rangle}{\bar{n}} \frac{1}{\mu^{q / 2} F}\right]^{-1} .
$$




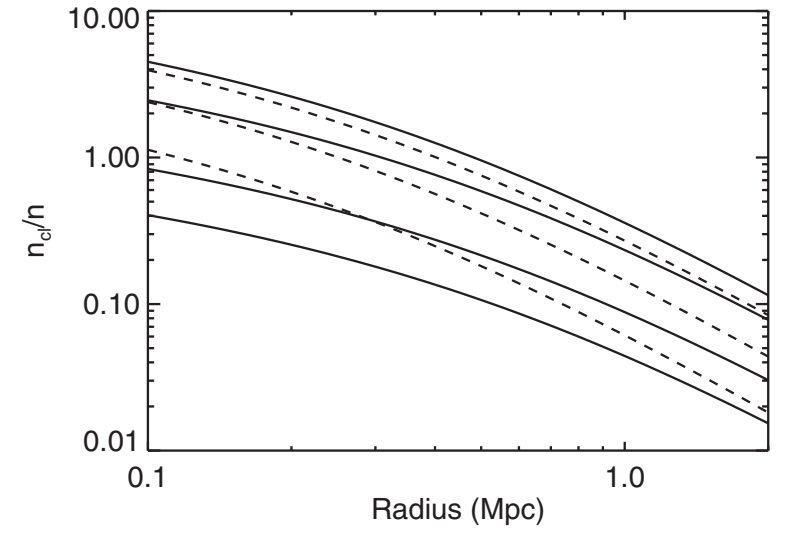

Figure 6. Top panel: the ratio $\overline{\mathrm{cl}_{\mathrm{cl}}} / \bar{n}$ of the projected density of cluster galaxies to the mean galaxy density of the survey for a halo of mass $M=10^{15} M_{\odot}$ for a variety of redshifts. From bottom to top, the redshift of the solid curves are $z=0.1,0.15,0.3$, and 0.6. From top to bottom, the redshift of the dashed lines are $z=0.9,1.2$, and 1.5 . The redshift slice $0.2 \lesssim z \lesssim 1.0$, the ratio $\overline{n_{\mathrm{cl}}} / \bar{n}$ can be larger than unity in the inner few hundred kpc for massive halos.

Here, $\left\langle n_{\mathrm{cl}}\right\rangle$ is the mean number density of cluster member galaxies in the stack considered. We find that $\left\langle\widehat{\Delta \Sigma}^{\prime}\right\rangle$ is sensitive to all three of our systematics parameters $r_{\mathrm{fg}}, r_{\mathrm{bg}}$, and $r_{\mathrm{cl}}$. While these parameters only appear as two independent ratios $-r_{\mathrm{fg}} / r_{\mathrm{bg}}$ and $r_{\mathrm{cl}} / r_{\mathrm{bg}}$-in practice we still need to estimate all three parameters independently.

Just how sensitive is $\widehat{\Delta \Sigma}^{\prime}$ to systematic uncertainties? As for $\widehat{\Delta \Sigma}$, we compute the relative systematic error due to a small uncertainty in our systematics parameters. Linearizing, we find

$$
\sigma_{\text {sys }}\left(\ln \Delta \Sigma^{\prime}\right)=-\delta r_{\mathrm{fg}} \frac{1-F}{\mu^{q / 2} F}-\delta r_{\mathrm{cl}} \frac{\left\langle n_{\mathrm{cl}}\right\rangle}{\bar{n}} \frac{1}{\mu^{q / 2} F} .
$$

Comparing the above expression to Equation (34), it is clear that $\widehat{\Delta \Sigma}^{\prime}$ is more robust to systematics if the coefficient of $\delta r_{\mathrm{fg}}$ and $\delta r_{\mathrm{cl}}$ are smaller than unity. Inspecting the above expression we notice two important things: first, the coefficients scale as $1 / F$, so at large redshifts, $\widehat{\Delta \Sigma}$ is mathematically guaranteed to become more robust than $\widehat{\Delta \Sigma}$, though whether this limit is reached in practice is unclear. Second, the coefficient for $\delta r_{\mathrm{cl}}$ scales as $n_{\mathrm{cl}} / \bar{n}$ (dilution effect), which we expect to be larger than unity in the cores of clusters, but insignificant in the outskirts, so it is possible for $\widehat{\Delta \Sigma}$ to be more robust than $\widehat{\Delta \Sigma}^{\prime}$ in the cores of clusters, but the opposite be true in the outskirts.

Figure 6 shows the ratio $\overline{n_{\mathrm{cl}}} / \bar{n}$ for a halo of mass $10^{15} M_{\odot}$ as a function of radius for a variety of redshifts. The ratio has been estimated using the model detailed in Appendix C. As we expected, this ratio is larger than unity in the inner few hundred $\mathrm{kpc}$ for $0.2 \lesssim z \lesssim 1.2$. Note, however, that this is true for very massive halos. If we assume instead $M=10^{14}$, this ratio barely reaches unity at $R=100 \mathrm{kpc}$. Note that the simple model for cluster members used here should only be taken as an order-ofmagnitude estimate.

Figure 7 shows the relative systematic error for both the $\widehat{\Delta \Sigma}$ and $\widehat{\Delta \Sigma}^{\prime}$ estimators assuming a systematic uncertainty $\delta r_{\mathrm{bg}}=\delta r_{\mathrm{fg}}=\delta r_{\mathrm{cl}}=1 \%$ (one can easily scale the values in Figure 7 to other values of $\delta r$ ). For the estimator $\widehat{\Delta \Sigma}$, the corresponding systematic error is constant, and mass and redshift are independent. The same is not true of $\widehat{\Delta \Sigma}^{\prime}$. We find that for massive halos, the error in $\widehat{\Delta \Sigma}^{\prime}$ is typically dominated by dilution of the lensing signal by cluster member galaxies.

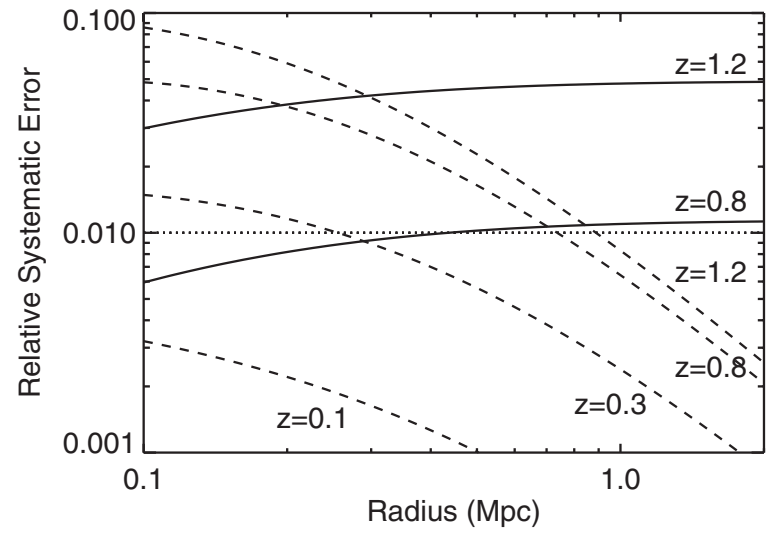

Figure 7. Relative systematic error for both $\widehat{\Delta \Sigma}$ (dotted) and $\widehat{\Delta \Sigma}^{\prime}$ (solid and dashed) as a function of radius for a halo of mass $M=10^{15} M_{\odot}$. The solid curves show the error on $\widehat{\Delta \Sigma}^{\prime}$ due to a $1 \%$ systematic uncertainty $\delta r_{\mathrm{fg}}$ on the fraction of foreground galaxies included in the source galaxy population (the curves for $z=0.1,0.3$ are below the minimum error plotted). Dashed curves are the relative systematic error on $\widehat{\Delta \Sigma}^{\prime}$ due to a $1 \%$ error on $r_{\mathrm{cl}}$, the fraction of cluster galaxies mistakenly included in the source population. Finally, the dotted curves are the (redshift-independent) relative error on $\widehat{\Delta \Sigma}$ due to a $1 \%$ systematic error $\delta r_{\mathrm{bg}}$ in the estimated fraction of background galaxies in the source population.

This error becomes larger than the expected statistical error in $\widehat{\Delta \Sigma}$ at $z \approx 0.3$, though it affects only the innermost few hundred kpc scales. Moreover, this error is explicitly mass dependent, with less massive halos benefiting from smaller systematic uncertainties. In all cases, however, for $z \gtrsim 0.8$, the $1 / F$ dependence of the systematic error on $\widehat{\Delta \Sigma}^{\prime}$ makes this estimator less robust than $\widehat{\Delta \Sigma}$.

From the above discussion alone, it is unclear if either of the two estimators we have considered here is superior to the other. At very high masses and high redshift, $\widehat{\Delta \Sigma}$ is likely to be preferable to $\widehat{\Delta \Sigma}^{\prime}$, but this may be reversed as one moves toward lower redshifts and/or masses. Furthermore, choosing between $\widehat{\Delta \Sigma}$ and $\widehat{\Delta \Sigma}^{\prime}$ also amounts to choosing between a spatially constant, mass-independent but likely redshift-dependent systematic in the case of $\widehat{\Delta \Sigma}$, and a significantly mass- and radius-dependent systematic in case of $\widehat{\Delta \Sigma}^{\prime}$. The cluster member dilution affecting $\widehat{\Delta \Sigma}^{\prime}$ is frequently corrected for by multiplying the shear profile measured with $\widehat{\Delta \Sigma}^{\prime}$ by the observed source galaxy-cluster correlation function (e.g., "boost factor" in Mandelbaum et al. 2005). This is essentially equivalent to using the estimator $\widehat{\Delta \Sigma}$ from the start. Finally, the answer as to which estimator is preferable will also depend on which of the systematic uncertainties discussed here $-r_{\mathrm{bg}}, r_{\mathrm{fg}}$, and $r_{\mathrm{cl}}-$ can be best controlled.

\subsection{Other Systematics}

In addition to the systematics discussed above, there are additional sources of systematic uncertainty that can impact our results. A few examples of such sources of systematic uncertainty are as follows.

1. Shear systematics. If shear is misestimated, this systematic will of course be carried over to the estimated cluster mass.

2. Miscentering. If clusters are miscentered, this can have a dramatic impact on the expectation value of the weak lensing signal in the cores of clusters (Johnston et al. 2007b). This systematic will almost certainly dominate the uncertainty with which the concentration of galaxy clusters 
can be measured within cluster stacks, but its impact on cluster masses can be significantly reduced through careful analysis (Mandelbaum et al. 2010).

3. Source obscuration by foreground galaxies. Occasionally, background galaxies will be perfectly aligned with foreground galaxies, and therefore the latter cannot be included in the weak lensing shear signal estimation. This reduces the effective area of the annuli used to estimate shear, which in turn impacts the expected number of galaxies in the annulus, thereby impacting globally normalized estimators.

4. Photometric redshift errors. The strength of the shear signal depends on the redshift of the source galaxy under consideration, which must be estimated based on photometric data. Consequently, scatter and catastrophic errors in photometric redshift estimates may have a significant impact on shear mass calibration experiments (see e.g., Mandelbaum et al. 2008b).

5. Baryonic effects. The inner profiles of galaxy clusters is expected to be significantly impacted by baryonic cooling, which is difficult to simulate in detail, and represent a theoretical systematic in our interpretation of stacked weak lensing profiles of galaxy clusters (see e.g., Gnedin et al. 2004).

In light of this discussion, we reiterate that determining whether $\widehat{\Delta \Sigma}$ is superior to $\widehat{\Delta \Sigma}^{\prime}$ or vice versa will require empirical investigation, and that due to these systematics, the forecasted precison for a DES-like survey is best interpreted as the lower limit for what will actually be realized. That said, until one estimator may be conclusively shown to be superior to the other, estimating cluster masses using both estimators should allow one to estimate the level of systematic uncertainty introduced by source galaxy selection, which should be of tremendous utility in upcoming photometric surveys.

\section{THE IMPACT OF STACKED WEAK LENSING ON CLUSTER ABUNDANCE EXPERIMENTS}

\subsection{Fisher Matrix and the Fiducial Model}

We now consider whether the statistical precision of the mean weak lensing masses recovered from stacked weak lensing is sufficient to significantly improve cosmological constraints in a DES-like survey relative to the self-calibration expectation with Planck priors. We address this question by once again relying on the Fisher matrix formalism. Specifically, we set the Fisher matrix for our experiment to the sum of the standard self-calibration result plus an additional contribution due to the stacked weak lensing data,

$$
F_{\text {total }}=F_{\text {Planck }}+F_{\mathrm{SC}}+F_{\mathrm{WL}} \text {. }
$$

The Planck priors Fisher matrix is that provided by $\mathrm{Hu} \& \mathrm{Ma}$ (private communication). For the self-calibration fisher matrix, we use the formalism described in detail in Wu et al. (2008) and $\mathrm{Wu}$ et al. (2010). Briefly, we assume that the survey area $5,000 \mathrm{deg}^{2}$ is divided into 500 patches of $10 \mathrm{deg}^{2}$ each. The observables in our experiment are the cluster counts in each of these 500 patches for each mass and redshift bin. We adopt the same binning as for the weak lensing analysis (five bins per decade in mass, and redshift slices of width $\pm \Delta z=0.05$ ).

The model parameters we consider can be split into two categories, cosmological parameters, and the nuisance parameters describing the observable-mass relation. The cosmological parameters and their fiducial values are given in the Introduction, except that we add as free parameters $w_{0}$ and $w_{a}$. These describe the equation of state of the dark energy $w=w_{0}+w_{a}(1-a)$ as a function of the cosmic expansion factor $a$. We will be primarily interested in how the Dark Energy Task Force (Albrecht et al. 2006) figure of merit - defined as the the product of the eigenvalues of the $w$ and $w_{a}$ Fisher matrix - changes upon inclusion of the weak lensing data.

In order to describe the observable-mass relation, each cluster is assumed to have an observed "mass" $M_{\text {obs }}$ which represents its richness measurement. We assume the observable-mass relation $P\left(M_{\mathrm{obs}} \mid M_{\text {true }}, z\right)$ is log-normal, with the mean and variance of $\ln M_{\mathrm{obs}}$ assumed to scale linearly with $\ln M$ and $\ln (1+z)$. We write

$$
\begin{gathered}
\left\langle\ln M_{\mathrm{obs}}\right\rangle=\ln M_{0}+\alpha_{M} \ln \left(\frac{M}{M_{\text {pivot }}}\right)+\alpha_{z} \ln (1+z) \\
\sigma_{\mathrm{obs}}^{2}=\sigma_{0}^{2}+\beta_{M} \ln \left(\frac{M}{M_{\text {pivot }}}\right)+\beta_{z} \ln (1+z) .
\end{gathered}
$$

We set $M_{\text {pivot }}=7 \times 10^{13} M_{\odot} \cdot{ }^{13}$ The remaining set of parameters are allowed to vary, and all of our results are marginalized over these observable-mass parameters. We set the fiducial value of our free parameters to those of an unbiased estimator in $\ln M_{\mathrm{obs}}$, so that $\ln M_{0}=\ln M$ and $\alpha_{M}=0$ and $\alpha_{z}=0$. Further, for our fiducial model we assume no evolution of the scatter with mass or redshift, so that $\beta_{M}=\beta_{z}=0$. We consider two possible values of the amplitude of the scatter $\sigma_{0}=0.2$ and $\sigma_{0}=0.5$, corresponding to $20 \%$ and $50 \%$ scatter in $M_{\text {obs }}$ at fixed $M_{\text {true }}$. The scatter in mass at fixed richness for maxBCG clusters is measured to be $45 \%$, though we note we expect to be able to reduce this scatter in the future (see e.g., Rozo et al. 2009b).

Our stacked weak lensing analysis allows us to introduce an additional set of observables in our analysis, namely the mean mass $M_{W L}$ in bins of $M_{\mathrm{obs}}$. Since the mean mass estimates of the different bins are independent, the weak lensing Fisher matrix is simply

$$
F_{\mathrm{WL}}=\sum_{\text {all bins }} \frac{1}{\sigma_{\mathrm{WL}}^{2}} \frac{\partial\left\langle\ln M_{\mathrm{WL}}\right\rangle}{\partial p_{i}} \frac{\partial\left\langle\ln M_{\mathrm{WL}}\right\rangle}{\partial p_{j}},
$$

where $\sigma_{\mathrm{WL}}$ is the error in the mass estimated in Section 2.4. To compute $\left\langle\ln M_{\mathrm{WL}}\right\rangle$, we assume that weak lensing masses are unbiased, so that

$$
\begin{gathered}
\left\langle M_{\mathrm{WL}} \mid M_{\mathrm{obs}}, z\right\rangle=\left\langle M_{\text {true }} \mid M_{\mathrm{obs}}, z\right\rangle \\
=\frac{1}{\bar{N}} \int d M_{\text {true }} d z M_{\text {true }} \frac{d n}{d M_{\text {true }}} \frac{d V}{d z}\langle\phi\rangle
\end{gathered}
$$

where $\phi$ is the binning function in observed mass and redshift, $\langle\phi\rangle$ is the effective binning function as a function of true halo mass,

$$
\left\langle\phi \mid M_{\text {true }}, z\right\rangle=\int d M_{\mathrm{obs}} \phi\left(M_{\mathrm{obs}}, z\right) P\left(M_{\mathrm{obs}} \mid M_{\mathrm{true}}, z\right),
$$

and $\bar{N}$ is the expected number of clusters,

$$
\bar{N}=\int d M_{\text {true }} d z \frac{d n}{d M_{\text {true }}} \frac{d V}{d z}\langle\phi\rangle .
$$

13 Note $\ln M_{0}$ and $\ln M_{\text {pivot }}$ are degenerate, so we can fix one of them arbitrarily without loss of generality. 


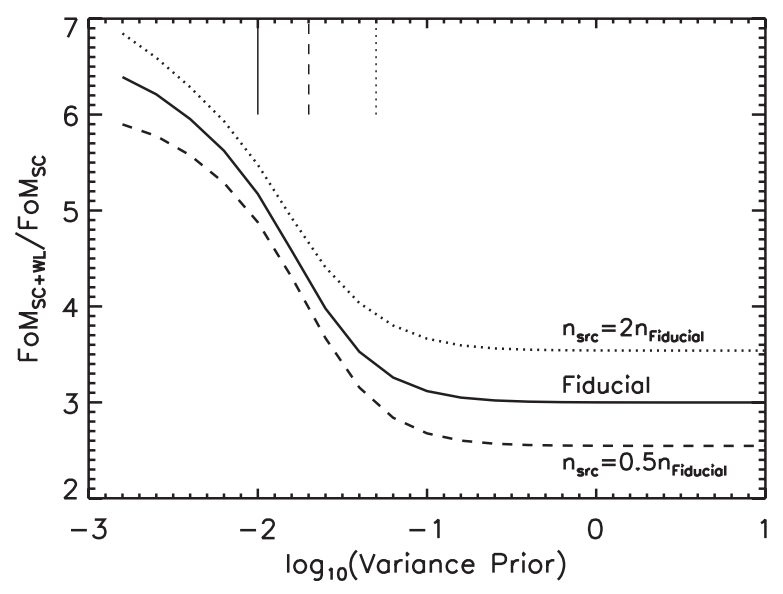

Figure 8. Figure of merit for a DES-like cluster abundance experiment with stacked weak lensing mass calibration relative to its self-calibration expectation, as function of the external prior $\Delta \sigma_{0}^{2}$ on the scatter in the mass-observable relation. Planck priors are assumed. The solid lines employ the forecasted errors arrived at in Section 2.4. The dashed and dotted lines have a source density that is a factor of two lower (dashed) and higher (dotted) than that of our fiducial model. The long tick marks along the top axis correspond to a $5 \%$ prior on the scatter assuming $\sigma_{0}=0.1$ (solid), $\sigma_{0}=0.2$ (dashed), and $\sigma_{0}=0.5$ (dotted). The relative improvement in the figure of merit is nearly independent of the adopted value of $\sigma_{0}$, particularly for broad priors.

A more detailed discussion of how to add this additional information to cluster forecasts is presented in Wu et al. (2010).

Before moving on, we point out that since the error estimates $\sigma_{\mathrm{WL}}$ of the weak lensing masses from Section 2.4 assumed clusters were binned according to their true masses, there is a small scatter-dependent correction to the predicted uncertainties. Given that we have not taken into account finite mass bin-width effects (see Section 2.3), we ignore these corrections in this section as well, and simply remind the reader that the corrections are expected to be small since shape noise is larger than the intrinsic scatter in the mass.

\subsection{Results}

Figure 8 shows the figure of merit of our DES-like survey including stacked weak lensing mass calibration relative to the figure of merit obtained without this additional source of data. We have allowed for the possibility of a prior on the scatter parameters with $\Delta \sigma_{0}^{2}=\Delta \beta_{M}=\Delta \beta_{z}$. The solid line assumes the weak lensing uncertainty for each mass and redshift bin $\sigma_{W L}$ estimated in Section 2.4, whereas the dashed and dotted assumed a source density that is half and twice that of our fiducial model respectively. Assuming no scatter priors, we find that the improvement in the Dark Energy Task Force figure of merit is a factor of 2.5-3.5. This is true for both the $\sigma_{0}=0.2$ and the $\sigma_{0}=0.5$ models: the relative improvement in the figure of merit is only weakly dependent on the assumed scatter, which is why we only show one set of curves in Figure 8 (those for $\sigma_{0}=0.5$ ). The fiducial value for the figure of merit is, of course, different. We have $F o M_{\mathrm{SC}}=19$ for $\sigma_{0}=0.2$, while $F o M_{\mathrm{SC}}=15$ for $\sigma_{0}=0.5$.

Additional priors on the scatter of the mass-observable relation may further increase the efficacy of stacked weak lensing mass calibration, leading to improvements in the figure of merit as large as a factor of eight. To do so, however, the priors need to be very tight. Note that we have expressed these priors in terms of the variance rather than the standard deviation. For reference, the long thin vertical tick marks a long the top $x$-axis in Figure 8 correspond to a $5 \%$ prior (i.e., $\Delta \sigma_{0}=0.05$ on the scatter, assuming $\sigma_{0}=0.1$ (solid), $\sigma_{0}=0.2$ (dashed), and $\sigma_{0}=0.5$ (dotted). Note that while strictly speaking we are only showing the relative improvement for the $\sigma_{0}=0.5$ case, the curves for $\sigma_{=} 0.1$ and $\sigma_{0}=0.2$ closely track that for $\sigma_{0}=0.5$. It is evident from the Figure that accurate knowledge of the scatter can lead to significant further improvement in the figure of merit of the experiment.

We have also considered how biases in the weak lensing masses could impact cosmological parameter estimation in order to assess the level at which systematics need to be controlled. Not surprisingly, we find that $\approx 2 \%$ biases in mass-which correspond to $\approx 1 \sigma$-result in $\approx 1 \sigma$ biases in the inferred cosmological parameters. Whether the recovered weak lensing masses can indeed be expected to be unbiased at the $2 \%$ level in real data, however, remains to be seen.

\section{SUMMARY AND CONCLUSIONS}

Weak lensing shear profiles and weak lensing peak finding tend to utilize the shear signal in different ways, with shear profile mass calibration often relying on locally normalized estimators $\left(\widehat{\Delta \Sigma}^{\prime}\right)$, and peak often finding relying on globally normalized estimators $(\widehat{\Delta \Sigma})$. We have used both of these estimators to predict the precision to which the mean mass of galaxy cluster stacks in a DES-like survey can be measured. We find that for moderate redshift clusters $(z \lesssim 0.6)$, the typical precision achieved is $\approx 2 \%$, with the two types of estimators having nearly identical statistical uncertainties. A companion paper, Schmidt \& Rozo (2011), investigates similar issues in the case of weak lensing peak finding.

We also considered three sources of systematic biases for these measurements. The first is magnification bias, which we find affects $\widehat{\Delta \Sigma}$ but not $\widehat{\Delta \Sigma}^{\prime}$. For the former, we find that including magnification bias corrections to the expectation value of $\widehat{\Delta \Sigma}$ is necessary to avoid significant systematic biases in both halo mass and concentration. We emphasize however that magnification bias corrections can be easily incorporated into the data analysis, so this is not a particularly worrisome systematic. Our results simply indicate that it is necessary to incorporate these corrections.

The second source of systematic uncertainty we considered are finite bin-width corrections, which affect both $\widehat{\Delta \Sigma}$ and $\widehat{\Delta \Sigma}^{\prime}$. Again, these corrections can in principle be explicitly included when analyzing data, and our investigation only addresses whether doing so is necessary for practical purposes. We find that for logarithmic bins $\pm \Delta \log _{10} R \lesssim 0.04$, the systematic bias in mass from finite bin-width corrections is less than $1 \%$. If one uses five bins per decade in radius $\left( \pm \Delta \log _{10} R=0.1\right)$, biases as large as $5 \%$ in mass are possible.

The final systematic we consider here is fluctuations in the number density of galaxies due to intrinsic clustering, which can affect the estimators through imperfect photometric redshift selections. Remarkably, we find that $\widehat{\Delta \Sigma}$ and $\widehat{\Delta \Sigma}^{\prime}$ are affected by this systematic in very different ways: $\widehat{\Delta \Sigma}$ is affected by the fraction of background galaxies missed by photometric redshift selection, whereas $\widehat{\Delta \Sigma}^{\prime}$ is affected by the fraction of foreground or cluster member galaxies that are included in the source population. Whether $\widehat{\Delta \Sigma}$ or $\widehat{\Delta \Sigma}^{\prime}$ is more robust depends on which of these systematics can be better controlled, a question which can only be empirically resolved. In either case, both estimators can be used to cross-check each other for the effects discussed here, due to their significantly different systematics. 
Finally, having estimated the precision with which the mean cluster mass of clusters stacks can be measured with a DESlike survey, we have investigated how this measurement would impact the cosmological parameter constraints of a DES-like cluster abundance experiment. We find that with our fiducial assumptions, the figure of merit of such an experiment improves by a factor of 2.5-3.5, with larger increases possible if priors on the scatter of the observable-mass relation can be derived from additional observations. Furthermore, the improvement in the figure of merit induced by weak lensing mass calibration is almost independent of the magnitude of this scatter and should thus apply to a wide range of mass proxies.

E.R. thanks Mike Jarvis, Matthew Becker, Jörg Dietrich, Scott Dodelson, Henk Hoekstra, Anja von der Linden, and Raul Jimenez for helpful discussions. The authors also thank Josh Frieman and Jörg Dietrich for comments on an earlier version of this manuscript which helped to significantly improve the presentation. E.R. is funded by NASA through the Einstein Fellowship Program, grant PF9-00068. H.W. is supported by the Gabilan Stanford Graduate Fellowship and the SLAC National Accelerator Laboratory. F.S. is supported by the Gordon and Betty Moore Foundation at Caltech.

\section{APPENDIX A}

\section{SOURCE CLUSTERING}

In the main section of this paper, we assumed source galaxies are randomly distributed in the sky. We now investigate whether source clustering can have an impact on our results. Source clustering implies that Equation (10) must be replaced by

$$
\left\langle\delta_{i} \delta_{j}\right\rangle=\delta_{i j} \frac{1}{\mu^{q / 2} \bar{n} \Delta \Omega}+\xi_{i j},
$$

where $\xi_{i j}$ is the projected galaxy-galaxy correlation function. Note that in general this term will correlate different radial bins, so that if $\widehat{\Delta \Sigma}_{\alpha}$ and $\widehat{\Delta \Sigma}_{\beta}$ are the estimators at radial bins $\alpha$ and $\beta$, the new term in the covariance matrix is given by

$$
C_{\alpha \beta}=\bar{\Sigma}_{c}^{2} \mu_{\alpha}^{q / 2} \mu_{\beta}^{q / 2} g_{\alpha} g_{\beta} V_{\alpha \beta}
$$

where

$$
V_{\alpha \beta}=\frac{1}{A_{\alpha} A_{\beta}} \sum_{i j} \Delta \Omega^{2} \xi_{i j} W_{i}^{\alpha} W_{j}^{\beta}
$$

and $A_{\alpha}$ and $A_{\beta}$ are the area of the annulus $\alpha$ and $\beta$ respectively. Note we have used the thin annulus approximation to set $\mu$ and $g$ constant within each annulus. Taking now the continuum limit, we arrive at

$$
V_{\alpha \beta}=\frac{1}{A_{\alpha} A_{\beta}} \int \frac{d^{2} l}{(2 \pi)^{2}} P(l)\left|W_{\alpha}^{*}(l) W_{\beta}(l)\right| .
$$

Before going any further, it is worth taking a second to compare Equation (A2) with Equation (12). Specifically, note that shape noise is explicitly dependent on the source density, whereas source clustering is not. This implies that at a sufficiently high source density, source clustering must dominate. On the other hand, source clustering scales as $g^{2}$, whereas shape noise scales as $\sigma_{e}^{2}$. More precisely, source clustering will be relevant if $g_{\alpha}^{2} V_{\alpha \alpha}$ is comparable to $\sigma_{e}^{2} / 2 \bar{n} A$. Thus, unless the source density is quite large, we expect shape noise to dominate. What follows is a quantitative confirmation of this expectation.
To do so, we need to begin by estimating $V_{\alpha \beta}$, which in turn requires that we compute the projected source galaxy power spectrum. Let then $n_{c o}(\boldsymbol{x})$ be the comoving galaxy density field. Assuming flatness, the corresponding projected galaxy density field is

$$
n(\vec{\theta})=\int d z n_{c o} \chi^{2} \frac{d \chi}{d z} H\left(z-z_{L}\right),
$$

where $H$ is a step function that selects only galaxies at redshift larger than the lens redshift of interest $z_{L}$. The fluctuations in the source density field are therefore given by

$$
\delta(\vec{\theta})=\int d \chi g(\chi) \delta_{g}(\boldsymbol{x}),
$$

where $\delta_{g}(\boldsymbol{x})$ is the three-dimensional galaxy density contrast, and

$$
g(\chi)=\frac{1}{\int d z f(z) H\left(z-z_{L}\right)} f(z)\left(\frac{d \chi}{d z}\right)^{-1} H\left(z-z_{L}\right) .
$$

Using Limber's approximation, the angular power spectrum of the source density field is related to the three dimensional galaxy power spectrum via

$$
P(l)=\int d \chi \frac{g^{2}(\chi)}{\chi^{2}} P_{3 D}(l / \chi, z) .
$$

All that remains is to specify the three-dimensional galaxy power spectrum. To do so, we assume a constant scaleindependent bias $b=1$ relative to the non-linear matter power spectrum $P_{m m}(\boldsymbol{k}, z)$, which we compute using Smith et al. (2003) as implemented in the CAMB package (Lewis et al. 2000).

Having determined the projected source power spectrum, we need to compute the mean power over the filter functions $W_{\alpha}$. We assume $W_{\alpha}$ is a top hat in radius going from $\log _{10} R \in$ $\left[\log _{10} R_{c}-\Delta, \log _{10} R_{c}+\Delta\right]$, with $\Delta=0.02$ as per our fiducial assumptions. The Fourier transform of $W_{\alpha}$ is therefore

$$
\begin{gathered}
W_{\alpha}(l)=\int d^{2} \theta W_{\alpha}(\theta) \exp (i \boldsymbol{l} \cdot \boldsymbol{\theta}) \\
=2 \pi \int_{\theta_{\min }}^{\theta_{\max }} d \theta \theta J_{0}(l \theta) \\
=\frac{2 \pi}{l^{2}}\left[u_{\max } J_{1}\left(u_{\max }\right)-u_{\min } J_{1}\left(u_{\min }\right)\right],
\end{gathered}
$$

where $u=R l / d_{A}$ and $d_{A}$ is the angular diameter distance to the redshift of the lens. We now have all the ingredients necessary for computing the source clustering error.

Figure 9 compares the ratio between the shape noise term used in the main section of the paper, and the diagonal contribution $C_{\alpha \alpha}$ to the covariance matrix due to source clustering. Note we plot the ratio of the errors (i.e., the square root of the variance) rather than the ratio of the variance. The ratio of the noise terms is estimated for halos of mass $M=10^{14} M_{\odot}$ (thin lines) and $M=10^{15} M_{\odot}$ (thick lines) for three different redshifts, $z=0.1$ (solid), $z=0.5$ (dashed), and $z=0.9$ (dotted). We can see that source clustering is always significantly smaller than the corresponding shape noise terms, so that it can be safely neglected in our forecast. It is worth remarking, however, that the ratio of these two noise terms is dependent on the assumed radial bin width, with broader bins leading to smaller ratios reflecting the decreased shape noise. Figure 9 assumes our fiducial bin 


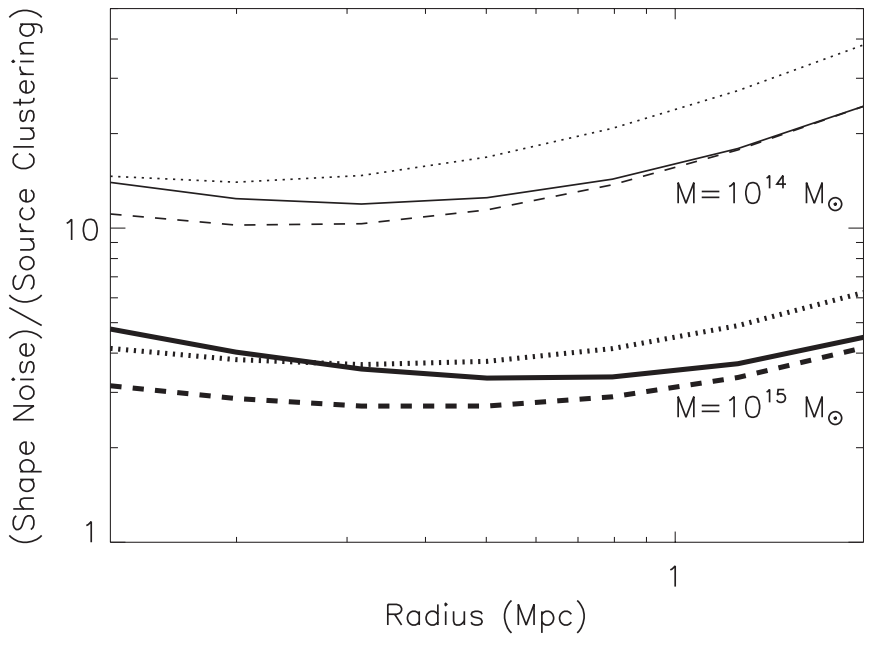

Figure 9. Ratio of the shape noise error considered in the main body of the manuscript to our estimated source clustering error using our fiducial assumptions for the estimator $\widehat{\Delta \Sigma}$. The ratio is shown for halos of mass $M=10^{14} M_{\odot}$ (thin lines) and $M=10^{15} M_{\odot}$. Different lines correspond to different redshifts, namely, $z=0.1$ (solid), $z=0.5$ (dashed), and $z=0.9$ (dotted). In all cases, we assume a concentration $c=5$. We conclude that for the source densities expected for the DES source clustering is a subdominant source of noise.

width $\pm \Delta \log _{10}=0.02$. If we were to use relative broad radial bins of width $\pm \Delta \log _{10} R=0.1$, the error ratio can drop to a factor of three for the $10^{15} M_{\odot}$ halos and down to a factor of $\sim 10$ for the $10^{14} M_{\odot}$ halos. Thus, even when employing broad radial bins, the errors are dominated by shape noise rather than by source clustering.

\section{APPENDIX B}

\section{THE MEAN AND VARIANCE OF $\widehat{\Delta \Sigma}^{\prime}$}

The estimator $\widehat{\Delta \Sigma}^{\prime}$ takes the form $\widehat{\Delta \Sigma}^{\prime}=\bar{\Sigma}_{c} x / y$, where

$$
\begin{gathered}
x=\sum_{i} \Delta \Omega\left(1+\delta_{i}\right) e_{i} W_{i} \\
y=\sum_{i} \Delta \Omega\left(1+\delta_{i}\right) W_{i} .
\end{gathered}
$$

We write $x=\bar{x}+\Delta x$ and $y=\bar{y}+\Delta y$, and use the binomial expansion to solve for $\widehat{\Delta \Sigma}^{\prime}$ assuming $\Delta y / \bar{y} \ll 1$ to expand up to second order. We find

$$
\widehat{\Delta \Sigma}^{\prime}=\Sigma_{c} \frac{\bar{x}}{\bar{y}}\left[1+\frac{\Delta x}{\bar{x}}-\frac{\Delta y}{\bar{y}}-\frac{\Delta x \Delta y}{\bar{x} \bar{y}}+\frac{\Delta y^{2}}{\bar{y}^{2}}\right] .
$$

Upon taking the expectation value, and using the fact that $\bar{x}=A g$ and $\bar{y}=A$, we arrive at $\left\langle\widehat{\Delta \Sigma}^{\prime}\right\rangle=\Sigma_{c} g$, as per Equation (23). Squaring Equation (B3), we find

$$
\left(\widehat{\Delta \Sigma}^{\prime}\right)^{2}=\Sigma_{c}^{2}\left(\frac{\bar{x}}{\bar{y}}\right)^{2}\left[1+\frac{\Delta x^{2}}{\bar{x}^{2}}+\frac{3 \Delta y^{2}}{\bar{y}^{2}}-\frac{4 \Delta x \Delta y}{\bar{x} \bar{y}}\right] .
$$

To compute the expectation value, we use the fact that $\bar{x}=g A$, $\bar{y}=A$, and

$$
\left\langle\Delta x^{2}\right\rangle=\left(g^{2}+\frac{1}{2} \sigma_{e}^{2}\right) \frac{A}{\mu^{q / 2} \bar{n}}
$$

$$
\begin{aligned}
& \langle\Delta x \Delta y\rangle=\frac{g A}{\mu^{q / 2} \bar{n}} \\
& \left\langle\Delta y^{2}\right\rangle=\frac{A}{\mu^{q / 2} \bar{n}} .
\end{aligned}
$$

The expectation value of $\left\langle\widehat{\Delta \Sigma}^{\prime 2}\right\rangle$ simplifies to

$$
\left\langle\left(\widehat{\Delta \Sigma}^{\prime}\right)^{2}\right\rangle=\Sigma_{c}^{2} g^{2}\left[1+\frac{1}{\mu^{q / 2} \bar{n} A} \frac{\sigma_{e}^{2}}{2 g^{2}}\right],
$$

which leads directly to Equation (24).

\section{APPENDIX C}

\section{A MODEL FOR CLUSTER GALAXIES}

In order to compute this systematic error due to $\delta r_{\mathrm{cl}}$ we must first estimate the ratio $\overline{n_{\mathrm{cl}}} / \bar{n}$. For this, we derive an order-ofmagnitude estimate as follows: let $\epsilon$ be the fraction of galaxies within a narrow redshift slice $\pm \Delta z$ about the redshift of the cluster, and $\pm L$ be the corresponding physical width of the slice. These two quantities are related to the redshift width of the slice via

$$
\epsilon=\frac{1}{\bar{n}} \frac{d \bar{n}}{d z} 2 \Delta z=\frac{f(z)}{\int_{0}^{\infty} d z^{\prime} f\left(z^{\prime}\right)} 2 \Delta z,
$$

where $f(z)$ is given by Equation (14), and

$$
L=\frac{\Delta z}{1+z} c H^{-1}
$$

Now, the mean three dimensional galaxy density $\rho_{g}$ within the redshift slice is

$$
\bar{\rho}_{g}=\frac{\epsilon \bar{n}}{2 L D_{A}^{2}} .
$$

Letting $\delta_{g}$ be the galaxy density contrast field, the projected cluster galaxy density is given by

$$
\begin{aligned}
\overline{n_{\mathrm{cl}}} & =D_{A}^{2} \int \frac{d \chi}{1+z} \rho_{g}=D_{A}^{2} \bar{\rho}_{g} \int \frac{d \chi}{1+z}\left(1+\delta_{g}\right) \\
& =\frac{\epsilon \bar{n}}{2 L} \int \frac{d \chi}{1+z}\left(1+\delta_{g}\right) .
\end{aligned}
$$

Assuming galaxies trace mass, we can set $\delta_{m}=\delta_{g}$, and therefore

$$
\left(1+\delta_{g}\right)=\left(1+\delta_{m}\right)=\frac{\rho_{m}}{\bar{\rho}_{m}(z)}
$$

Inserting this into our expression for $\overline{n_{\mathrm{cl}}}$,

$$
\overline{n_{\mathrm{cl}}}=\frac{\epsilon \bar{n}}{2 L} \int \frac{d \chi}{1+z} \frac{\rho_{m}}{\bar{\rho}_{m}\left(z_{L}\right)}=\frac{\epsilon \bar{n}}{2 L \bar{\rho}_{m}(z)} \Sigma .
$$

Inserting our expressions for $\epsilon$ and $L$, and setting $\bar{\rho}_{m}=$ $\Omega_{m} \rho_{c}(1+z)^{3}$ we finally arrive at

$$
\frac{\overline{n_{\mathrm{cl}}}}{\bar{n}}=\frac{f(z)}{\int_{0}^{\infty} d z^{\prime} f\left(z^{\prime}\right)} \frac{\Sigma}{(1+z)^{2} \Omega_{m} \rho_{c} c H^{-1}(z)} .
$$




\section{REFERENCES}

Albrecht, A., et al. 2006, arXiv:astro-ph/0609591

Bartelmann, M. 1996, A\&A, 313, 697

Bernardeau, F. 1998, A\&A, 338, 375

Cunha, C. 2009, Phys. Rev. D, 79, 063009

Cunha, C., Huterer, D., \& Frieman, J. A. 2009, Phys. Rev. D, 80, 063532

Faltenbacher, A., Li, C., Mao, S., van den Bosch, F. C., Yang, X., Jing, Y. P., Pasquali, A., \& Mo, H. J. 2007, ApJ, 662, L71

Gladders, M. D., Yee, H. K. C., Majumdar, S., Barrientos, L. F., Hoekstra, H., Hall, P. B., \& Infante, L. 2007, ApJ, 655, 128

Gnedin, O. Y., Kravtsov, A. V., Klypin, A. A., \& Nagai, D. 2004, ApJ, 616, 16

Henry, J. P., Evrard, A. E., Hoekstra, H., Babul, A., \& Mahdavi, A. 2009, ApJ, 691, 1307

Hoekstra, H. 2003, MNRAS, 339, 1155

Hu, W., \& Cohn, J. D. 2006, Phys. Rev. D, 73, 067301

Huterer, D., \& Linder, E. V. 2007, Phys. Rev. D, 75, 023519

Johnston, D. E., Sheldon, E. S., Tasitsiomi, A., Frieman, J. A., Wechsler, R. H., \& McKay, T. A. 2007a, ApJ, 656, 27

Johnston, D. E., et al. 2007b, arXiv:0709.1159

Koester, B. P., et al. 2007, ApJ, 660, 239

Leauthaud, A., et al. 2010, ApJ, 709, 97

Lewis, A., Challinor, A., \& Lasenby, A. 2000, ApJ, 538, 473

Lima, M., \& Hu, W. 2004, Phys. Rev. D, 70, 043504

Lima, M., \& Hu, W. 2005, Phys. Rev. D, 72, 043006

Lombriser, L., Slosar, A., Seljak, U., \& Hu, W. 2010, arXiv:1003.3009

Majumdar, S., \& Mohr, J. J. 2003, ApJ, 585, 603

Majumdar, S., \& Mohr, J. J. 2004, ApJ, 613, 41

Mandelbaum, R., Seljak, U., Baldauf, T., \& Smith, R. E. 2010, MNRAS, 683

Mandelbaum, R., Seljak, U., \& Hirata, C. M. 2008a, J. Cosmol. Astropart. Phys., JCAP08(2008)006

Mandelbaum, R., et al. 2005, MNRAS, 361, 1287
Mandelbaum, R., et al. 2008b, MNRAS, 386, 781

Mantz, A., Allen, S. W., Ebeling, H., \& Rapetti, D. 2008, MNRAS, 387, 1179

Medezinski, E., et al. 2007, ApJ, 663, 717

Mortonson, M. J., Hu, W., \& Huterer, D. 2010, Phys. Rev. D, 81, 063007

Navarro, J. F., Frenk, C. S., \& White, S. D. M. 1996, ApJ, 462, 563

Oguri, M., \& Takada, M. 2011, Phys. Rev. D, 83, 023008

Rapetti, D., Allen, S. W., Mantz, A., \& Ebeling, H. 2010, MNRAS, 406, 1796

Rozo, E., \& Schmidt, F. 2010, arXiv:1009.5735

Rozo, E., et al. 2007, arXiv:astro-ph/0703571

Rozo, E., et al. 2009a, ApJ, 699, 768

Rozo, E., et al. 2009b, ApJ, 703, 601

Rozo, E., et al. 2010, ApJ, 708, 645

Schmidt, F., \& Rozo, E. 2011, ApJ, 735, 119

Schmidt, F., Rozo, E., Dodelson, S., Hui, L., \& Sheldon, E. 2009a, ApJ, 702, 593

Schmidt, F., Rozo, E., Dodelson, S., Hui, L., \& Sheldon, E. 2009b, Phys. Rev. Lett., 103, 051301

Schmidt, F., Vikhlinin, A., \& Hu, W. 2009c, Phys. Rev. D, 80, 083505

Schneider, P., \& Seitz, C. 1995, A\&A, 294, 411

Sealfon, C., Verde, L., \& Jimenez, R. 2006, ApJ, 649, 118

Shapiro, C., Dodelson, S., Hoyle, B., Samushia, L., \& Flaugher, B. 2010, Phys. Rev. D, 82, 043520

Sheldon, E. S., et al. 2009, ApJ, 703, 2217

Smith, R. E., et al. 2003, MNRAS, 341, 1311

Tinker, J., Kravtsov, A. V., Klypin, A., Abazajian, K., Warren, M., Yepes, G., Gottlöber, S., \& Holz, D. E. 2008, ApJ, 688, 709

Vallinotto, A., Dodelson, S., \& Zhang, P. 2010, arXiv:1009.5590

Vikhlinin, A., et al. 2009, ApJ, 692, 1033

White, M., Cohn, J. D., \& Smit, R. 2010, MNRAS, 408, 1818

Wright, C. O., \& Brainerd, T. G. 2000, ApJ, 534, 34

Wu, H., Rozo, E., \& Wechsler, R. H. 2010, ApJ, 713, 1207

Wu, H.-Y., Rozo, E., \& Wechsler, R. H. 2008, ApJ, 688, 729 